McBeck, J., Mair, K., Renard, F. (2018) Linking macroscopic failure with micromechanical processes in layered rocks: How layer orientation and roughness control macroscopic behavior. Tectonophysics, https://doi.org/10.1016/j.tecto.2018.11.016.

\title{
Linking macroscopic failure with micromechanical processes in layered rocks: How layer orientation and roughness control macroscopic behavior
}

\author{
Jessica McBeck ${ }^{1}$, Karen Mair ${ }^{1,2}$, François Renard $^{1,3}$
}

1 Physics of Geological Processes, The Njord Centre, Department of Geosciences, University of Oslo, Norway

2 currently on sabbatical at: School of Geosciences, University of Edinburgh, Scotland 3 University Grenoble Alpes, University Savoie Mont Blanc, CNRS, IRD, IFSTTAR, ISTerre, 38000 Grenoble, France

Corresponding author

Jessica McBeck

j.a.mcbeck@geo.uio.no 


\section{Abstract}

2 To constrain the impact of preexisting mechanical weaknesses on strain localization culminating

3 in macroscopic shear failure, we simulate triaxial compression of layered sedimentary rock using

4 three-dimensional discrete element method simulations. We develop a novel particle packing

5 technique that builds layered rocks with preexisting weaknesses of varying orientations,

6 roughness, and surface area available for slip. We quantify how the geomechanical behavior,

7 characterized by internal friction coefficient, $\mu_{0}$, and failure strength, $\sigma_{\mathrm{F}}$, vary as a function of

8 layer orientation, $\theta$, interface roughness, and total interface area. Failure of the simulated

9 sedimentary rocks mirrors key observations from laboratory experiments on layered sedimentary

10 rock, including minima $\sigma_{\mathrm{F}}$ and $\mu_{0}$ for layers oriented at $30^{\circ}$ with respect to the maximum

11 compressive stress, $\sigma_{1}$, and maxima $\sigma_{\mathrm{F}}$ and $\mu_{0}$ for layers oriented near $0^{\circ}$ and $90^{\circ}$ to $\sigma_{1}$. The

12 largest changes in $\sigma_{\mathrm{F}}(66 \%)$ and $\mu_{0}(20 \%)$ occur in models with the smoothest interfaces and

13 largest interface area. Within the parameter space tested, layer orientation exerts the most

14 significant impact on $\sigma_{\mathrm{F}}$ and $\mu_{0}$. These simulations allow directly linking micromechanical

15 processes observed within the models to macroscopic failure behavior. The spatial distributions

16 of nucleating microfractures, and the rate and degree of strain localization onto preexisting

17 weaknesses, rather than the host rock, are systematically linked to the distribution of failure

18 strengths. Preexisting weakness orientation more strongly controls the degree and rate of strain

19 localization than the imposed confining stress within the explored parameter space. Using the

20 upper and lower limits of $\mu_{0}$ and $\sigma_{\mathrm{F}}$ obtained from the models, estimates of the Coulomb shear

21 stress required for failure of intact rock within the upper seismogenic zone $(7 \mathrm{~km})$ indicates that a

22 rotation of $30^{\circ}$ of $\sigma_{1}$ relative to the weakness orientation may reduce the shear stress required for

23 failure by up to $100 \mathrm{MPa}$. 
25 Keywords: Anisotropy; preexisting weakness; layered rock; internal friction; failure strength;

26 discrete element method 


\section{Introduction}

28 Natural rocks contain mechanical weaknesses that span several orders of magnitude in scale,

29 from grain contacts to plate boundary faults. In particular, sedimentary basins host layers of

30 sediments and sedimentary rocks at various stages of deposition, burial, diagenesis and

31 lithification that may provide mechanical weaknesses along their contacts. Bedding-parallel

32 fractures between strata (e.g., Gale et al., 2014), and bedding-perpendicular fractures that

33 terminate at layer contacts (e.g., Pollard \& Aydin, 1988) demonstrate that interfaces between

34 sedimentary strata can act as mechanical weaknesses that influence stresses, localize strain and

35 impact macroscopic failure.

36 However, many numerical models of crack growth and fault development assume

37 homogeneous and isotropic host rock properties due, in part, to the scarcity of field and

38 laboratory data that constrain precisely how preexisting weaknesses impact the macroscopic

39 geomechanical behavior of the host rock. To constrain the influence of preexisting mechanical

40 weaknesses on macroscopic failure, we execute series of triaxial compression tests on discrete

41 element method simulations of layered sedimentary rock. We designed a new DEM layer

42 construction technique in order to capture mechanical anisotropy that arises from differences in

43 lithology and unconformities in layered sequences. We vary the layer orientation with respect to

44 the maximum compression direction, the layer interface roughness, the total layer interface area

45 available for slip, and the applied confining stress. We assess the impact of each parameter on

46 the concentration of microscopic failure along the preexisting weaknesses relative to diffuse

47 deformation throughout the host rock, and on the resulting macroscopic failure stress and internal

48 friction. Although previous experiments have investigated the impact of layer orientation on

49 failure strength (e.g., Duveau et al., 1998), this numerical analysis is the first to compare the 
50 relative impact of each of the aforementioned parameters on failure behavior, and the first to

51 investigate differences produced by layer interface roughness and area. The new layer

52 construction technique is critical to this parameterization. Furthermore, we directly link the

53 spatial distribution of microfracturing and strain localization to macroscopic geomechanical

54 response, thus providing new insights not yet available experimentally.

\section{2. Influence of mechanical weaknesses on failure}

56 Due to the prevalence of materials containing mechanical weaknesses, many experimentalists

57 have worked to understand how mechanical weaknesses impact rock failure and elastic

58 properties (e.g., Duveau et al., 1998). Many experiments have focused on the peak failure

59 strength of materials (e.g., Donath, 1961, 1964; Nova and Zaninetti, 1990; Shea and Kronenberg,

60 1993; Duveau et al., 1998; Cho et al., 2012; Fjær and Nes, 2014). For example, increasing mica

61 content in foliated gneiss decreases the compressional strength because biotite grains provide

62 nucleation sites for tensile microcracks (Rawling et al., 2002). Similarly, compression tests on

63 Mancos shale indicate that bedding planes are weak relative to the host rock and tend to fail

64 preceding macroscopic failure (Fjær and Nes, 2014). Here, we aim to capture differences in

65 mechanical behavior that arise in layered sedimentary rock.

66 Internal friction is an empirical term that experimentalists identify from the slope of linear

67 regression fits through experimental measurements of the stress conditions at or immediately

68 preceding failure (e.g., Byerlee, 1978). The internal friction and peak failure stress provide

69 estimates of the stress state at which rocks fail, and so are critical in robust predictions of brittle

70 failure within intact material at varying crustal conditions. Although many experiments have

71 identified relationships between the orientation of preexisting mechanical weaknesses and failure

72 stress (e.g., Shea and Kronenberg, 1993; Tavallali and Vervoort, 2010), few have attempted to 
73 constrain the impact of planar mechanical weaknesses on the internal friction of the host rock

74 (e.g., Donath, 1961; Duveau et al., 1998). This gap in understanding arises from the necessity of

75 breaking multiple rock cores at varying confining stresses in order to identify a relationship

76 between an applied confining stress and resulting failure stress, and so estimate internal friction.

77 Consequently, to robustly constrain internal friction and hence predict the conditions of brittle

78 rock failure at depth, experimentalists must use rock samples that are sufficiently similar to each

79 other so that the suite of experiments at varying confining stresses mimic the same characteristic

80 rock core. In addition, to characterize how microstructures are evolving and localizing with

81 accumulated strain requires labor-intensive serial experimentation in which tests on similar rock

82 cores are stopped at systematic strain increments (e.g., Mair et al., 2000). These tasks become

83 increasingly challenging when focusing on rocks with well-developed mechanical anisotropy.

84 In contrast to laboratory experiments, numerical simulations allow the deformation of

85 identical simulated rock containing mechanical weaknesses under a range of loading conditions.

86 Consequently, the natural variability between individual rock cores that often plague

87 experimental work does not have a strong influence on resulting geomechanical behavior in

88 numerical models. Furthermore, numerical methods enable systematic comparison of mechanical

89 properties of material with differing expressions of heterogeneity (e.g., Hentz et al., 2004;

90 Belheine et al., 2009; Scholtès \& Donzé, 2012, 2013; Dinç \& Scholtès, 2018), such as layers

91 with differing orientations and roughness, as in this study. Numerical models provide new

92 insights into how evolving microstructures control macroscopic behavior (e.g., Schöpfer et al.,

93 2009) that are generally not available in situ from laboratory experiments, unless the

94 experimentalists use techniques such as recording acoustic emissions (e.g., Stanchits et al., 2006)

95 or acquiring tomograms (e.g., Renard et al., 2018), from which they infer the evolving fracture 
96 network. Post-deformation procedures required after laboratory triaxial experiments, such as

97 unloading and epoxy impregnation for microscopic analyses, may alter or overprint the

98 microstructures. These procedures are not necessary for assessing microstructural evolution

99 within numerical models, and so deciphering such microstructural artifacts is not necessary.

100 3. Methods

101 To constrain the impact of preexisting mechanical weaknesses on macroscopic

102 geomechanical properties, we simulate triaxial compression of layered sedimentary rock using

103 the discrete element method modeling tool ESyS-Particle. We briefly introduce the underlying

104 physics of this tool. Then we describe the details of the models used in this study, including the

105 model loading conditions, model microparameters, calibration, and a novel particle packing

106 technique to construct the sedimentary layers.

107 3.1. ESyS-Particle

108 We triaxially compress simulated layered sedimentary rock using the parallel 3D discrete 109 element method (DEM) simulation package ESyS-Particle (Abe et al., 2003). Similar to other

110 DEM approaches (e.g., Cundall and Strack, 1979), ESyS-Particle simulates rock with many

111 individual spherical particles that are initially connected by elastic bonds that may fail following

112 a tensile criterion or the Coulomb shear failure criterion. The underlying physics implemented in

113 ESyS-Particle and other DEM implementations, such as YADE, are similar, although details of

114 the packing algorithms, contact laws and failure criteria differ.

115 ESyS-Particle simulations of fault gouge production produce realistic evolutions of fault

116 gouge size and sliding friction (Abe and Mair, 2005, 2009; Mair and Abe, 2008). The 3D

117 implementation of ESyS-Particle enables out-of-plane motion, which has proven important to

118 fault gouge production (Hazzard and Mair, 2003; Abe and Mair, 2005; Mair and Hazzard, 2007). 
119 These DEM simulations provide additional information beyond that available from laboratory

120 experiments, such as directed force networks, at fine spatiotemporal resolution throughout

121 deformation (e.g., Mair and Hazzard, 2007).

122 3.2. Construction of layered rock

123 To simulate triaxial compression tests of layered sedimentary rock using ESyS-Particle, we

124 build particle assemblages containing layers that are separated by preexisting weaknesses,

125 representing layered sedimentary rock. We build sample geometries of blocks (cuboids) with

126 length to width ratio $2: 1$ and cubes (ratio 1:1). We investigate both cubes and blocks because the

127 model shape influences the total layer interface area available for slip, and in particular, how

128 many bonds connect particles within layers (i.e., host rock matrix) and between layers (i.e.,

129 interfaces) for each layer orientation. Comparing the results from these sets of models highlights

130 the influence of the total layer interface area available for slip on rock failure. The dimensions of

131 the cubes are $20 \mathrm{~mm}$, while the dimensions of the rectangular blocks are $20 \mathrm{~mm}, 20 \mathrm{~mm}$ and 40

$132 \mathrm{~mm}$, with the long axis oriented vertically, parallel to $\sigma_{1}$ (Fig. 1). The model dimensions

133 constrain the choice of layer thickness $(5 \mathrm{~mm})$. This thickness allows the macroscopic failure

134 behavior to be independent of the specific particle packing geometry of one or two layer

135 interfaces because models include at least 4 layers. Here, we do not assess the influence of layer

136 thickness on failure behavior because this influence likely scales with the model volume, and so

137 may not yield significant insights into crustal deformation. In particular, the failure of a larger

138 model with thicker layers may be similar to the failure of a smaller model with thinner layers if

139 the total interface area available for slip are similar. In order to assess the influence of total

140 interface area of failure behavior, we change the shape of the model rather than the thickness of

141 the layers. 
142 To assess the influence of preexisting weaknesses on internal friction, we vary the orientation

143 of layers, and keep the direction of maximum compression constant (vertical). We measure layer

144 orientation, $\theta$, as the clockwise angle from the top side of the model to the layer interface (Fig.

145 1), which is also the angle between $\sigma_{1}$ and the normal to the layer interface. Horizontal layers

146 have $\theta=0^{\circ}$, and vertical layers have $\theta=90^{\circ}$. The layers are parallel in the z-dimension, which is

147 the out of plane direction.

148 We develop a novel particle packing technique in order to build layers of varying interface

149 roughness. We construct polygonal volumes and then fill the volumes with spherical particles

150 with radii of $0.1 \mathrm{~mm}$ to $1.0 \mathrm{~mm}$ (Fig. S1). We fill the volumes with particles starting from the

151 bottom volume and working upward, or in the case of vertical layers, from left to right. We fill

152 each volume using a standard particle packing technique of ESyS-Particle that has produced

153 DEM models that match the macroscopic behavior of brittle elastic materials. Then we connect

154 neighboring particles inside each layer with bonds, as well as the particles between each layer.

155 The variability in particle packing, and hence porosity, across layer interfaces are small. In order

156 to capture differences in mechanical strength along layer interfaces, we set the cohesion of bonds

157 within layers (i.e., host rock) to $20 \mathrm{MPa}$ and set the cohesion of bonds between layers (i.e.,

158 preexisting weaknesses) to $10 \mathrm{MPa}$. Calibration of the numerical failure strengths to

159 experimental strengths constrain the cohesion of bonds within the layers. The precise ratio of the

160 strength of the host rock and interfaces remains one of the least constrained by experimental

161 data. In addition, the effective strength of the interfaces arises from both the chosen cohesion and

162 particle packing across layer interfaces, as discussed below.

163 In ESyS-Particle, the bond tensile strength is determined from the microparameter Mohr-

164 Coulomb criterion constructed from the bond internal friction and cohesion. Extension of the 
165 criterion into the tensile normal stress regime determines the bond tensile strength. ESyS-Particle

166 allows the application of a truncation factor to reduce the tensile strength from that predicted by

167 the Mohr-Coulomb criterion, but we did not employ this factor here. Consequently, the

168 advantage of parameterizing the cohesion rather than a different microparameter is that doing so

169 changes both the tensile and shear failure criterions. The microparameters of bonds within each

170 layer are identical. The bonds within layers are stronger than the bonds between layers, and are

171 subsequently referred to as strong and weak bonds, respectively. The lack of employed

172 truncation factor may produce larger tensile macroscopic strengths than natural sedimentary

173 rocks, and so amplify the influence of shear failure relative to tensile failure.

174 To control the initial roughness of the layer interfaces (Fig. 2), we change the potential

175 spatial overlap of the adjacent layers (Fig. S1). This overlap parameter specifies how many

176 millimeters by which the boundaries of neighboring polygonal volumes overlap. With an overlap

177 of $1 \mathrm{~mm}$, for example, the vertical position of the bottom of a horizontal layer is $1 \mathrm{~mm}$ lower

178 than the vertical position of the top of the previous (lower) horizontal layer (Fig. S1). With

179 increasing overlap, particles are more closely packed along layer interfaces, and produce greater

180 roughness (Fig. S1, Fig. 2). The bottom surface of each layer interface is smoother than the top

181 surface because we pack the models starting from the bottom of the model (Fig. S1, Fig. 2).

182 A key question is whether the effective strengths of the simulated layer interfaces are

183 comparable to the strengths of layer interfaces in natural sedimentary strata, or other mechanical

184 weaknesses found in the crust. Both the bond cohesion and packing geometry along layer

185 interfaces influence the effective strength of the layer interface. However, the packing geometry

186 appears to exert a stronger influence than the bond cohesion. With zero grain overlap and no

187 applied confining stress, some models fail immediately after the particles experience 
188 gravitational forces because the particles do not geometrically interlock across the layer

189 interfaces. This lack of interlocking produces exceptionally smooth surfaces that provide little 190 resistance to gravity.

191 The slip-parallel and slip-perpendicular root-mean-squared roughness, $\chi_{\mathrm{RMS}}$, of slip surfaces

192 acquired from white light interferometer measurements (Renard et al., 2012) constrain the

193 potential range of $\chi_{\text {RMS }}$ of intact sedimentary interfaces (Fig. S2). Although the $\chi_{\mathrm{rms}}$ of intact

194 interfaces are likely higher than the $\chi_{\text {RMS }}$ of slipped surfaces, we expect that the slip-

195 perpendicular and slip-parallel roughness are on the same order of magnitude of the intact 196 interfaces. For example, in clay-rich sandstone and shales with a mean grain size, $G$, the finest 197 roughness is expected on to be on the scale of approximately $0.5 G$. However, there may be 198 random pitting on the order of a few millimeters due to local differential compaction or 199 displacements of grains during sedimentation. In the numerical models, we measure $\chi_{\mathrm{RMS}}$ by 200 identifying the layers of particles that connect adjacent layers, rotating the exposed layer contacts 201 so that they trend horizontal, and finding the elevation of each particle from the particle radius 202 and the y-coordinate (vertical) of the particle centroid. The resulting surface of particle 203 elevations reflects the roughness of the layer contact. The overlap distances of $0.2 \mathrm{~mm}$ and 0.5 $204 \mathrm{~mm}$ produce layer interfaces with $\chi_{\mathrm{RMS}}$ that overlap the ranges of the laboratory measurements of 205 slip-perpendicular and slip-parallel $\chi_{\text {RMS }}$ (Fig. S2).

206 With this particle packing technique, the particle radii range $(0.1-1.0 \mathrm{~mm})$ limits the 207 maximum $\chi_{\text {RMS }}$ achievable in the models, similar to the grain size in sedimentary rock. When the 208 overlap is greater than $0.5 \mathrm{~mm}$, the $\chi_{\mathrm{RMS}}$ remains relatively constant because increasing the 209 overlap does not allow denser particle packing between layers with the selected particle radii 
210 range. Despite these constraints, the $\chi_{\mathrm{RMS}}$ produced along the numerical layer interfaces

211 compares favorably with physical measurements of $\chi_{\mathrm{RMS}}$, lending confidence to the ability of the

212 models to capture the onset of macroscopic failure in layered sedimentary rock. These estimates

213 of roughness with $\chi_{\text {RMS }}$ may be used to estimate the corresponding joint roughness coefficient

214 (JRC) using empirical statistical relationships between JRC and $\chi_{\text {RMS }}$ (e.g., Tse \& Cruden, 1979).

215 This particle packing technique produces models with porosities that differ by $<1-2 \%$. The

216 porosity of models with layers with both overlap values range from $24-25 \%$, and models with no

217 layers have $23 \%$ porosity. Consequently, the variability in porosities between models is

218 analogous to natural variability.

219 Previous numerical studies have assessed anisotropy with DEM models using numerical

220 objects that behave as joints (e.g., Scholtès and Donzé, 2012). One central difference between

221 our implementation of anisotropy and these methods is that our approach enables modifying the

222 roughness of preexisting weaknesses. Consequently, our approach is more applicable to

223 sedimentary rocks constructed from the sediment deposition in which sediments and clays

224 gravitationally settle on top of older sediments, rather than jointed rock masses, for example.

\section{3.3. Calibration}

226 To simulate laboratory triaxial compression tests, we first apply linearly increasing confining

227 stress, from zero to the desired stress, on each of the six sides of the model such that $\sigma_{1}=\sigma_{2}=\sigma_{3}$.

228 Next, we displace the top and bottom sides synchronously at a constant velocity $(0.125 \mathrm{~mm} / \mathrm{s})$

229 toward the center of the model, increasing $\sigma_{1}$ while maintaining $\sigma_{2}=\sigma_{3}$. Once the maximum

230 desired loading velocity is reached, we continue to apply this velocity for several thousand time

231 steps until after the sample fails. 
The low applied velocity, linear ramp of the confining stress and loading velocity, and the

233 symmetrically loading of the top and bottom edges, enhances stability of the particle assemblage

234 by ensuring quasi-static conditions. In addition to these precautions, we calculate an appropriate

235 model time step length following the Courant condition (Courant et al., 1928), where the

236 numerically stable time step, $d_{t}$, is related the minimum particle mass, $M_{\min }$, and maximum

237 bond stiffness, $K_{\max }$, as

$238 \quad d_{t}<0.1 \sqrt{\frac{M_{\min }}{K_{\max }}}$

Eq. 1

239 Systematic tests conducted with varying bond and particle parameters (Table S1) produce

240 uniaxial compressive strengths (40-120 MPa) within the range of natural layered shale (e.g., Cho

241 et al., 2012).

242 Table S1 shows the values of microparameters that were constrained by careful calibration.

243 Pairs of bonded and unbonded particles have frictional and elastic interactions. We calculate the

244 corresponding particle density using the initial porosity of the model, $\phi(25 \%)$, and a desired bulk

245 density of $2700 \mathrm{~kg} / \mathrm{m}^{3}, \rho$, such that the particle density is $\rho(1-\phi)$. We then follow the established

246 numerical technique of increasing the particle density by a factor of $10^{6}$, which enables larger

247 time step lengths and thus shorter run times. Following the Courant condition (Eq. 1), the

248 minimum particle radius and maximum bond stiffness determine the time step length. The ramp

249 up times are sufficiently long to produce stable models in which the total kinetic energy does not

250 increase unbounded. Particles interact with the walls that apply the constant velocity or force

251 boundary conditions with elastic interactions following the prescribed stiffness, and without

252 frictional interactions. The tensile strength of the bonds is determined by extrapolation of the

253 Mohr-Coulomb failure criterion using the bond microparameters of internal friction and

254 cohesion. The microparameters of the bonded and unbonded particles are not expected to equal 
255 physical values of those properties. Instead, we calibrate the microparameters to produce bulk

256 macroscopic properties of the models that are within the range of physical measurements.

257 The failure of these simulated sedimentary rocks mirrors key observations from laboratory

258 experiments, including: failure envelopes that decrease in slope at higher confining stresses, $\sigma_{2}$,

259 broadening loading curves near peak stresses at higher $\sigma_{2}$, and smaller stress drops at higher $\sigma_{2}$.

260 Similarly consistent with laboratory experiments, $\sigma_{\mathrm{F}}$ and $\mu_{0}$ are minimized for layers oriented at

$26130^{\circ}$ with respect to $\sigma_{1}$, and maximized for layers oriented near $0^{\circ}$ and $90^{\circ}$ to $\sigma_{1}$. These

262 observations provide further evidence of robust calibration, and applicability of the numerical

263 results to crustal behavior. These observations are discussed in greater detail in the next section.

264 Furthermore, these loading conditions, microparameters and particle packing technique

265 produce models with bulk geomechanical responses that are not sensitive to location of

266 individual particles (Fig. S3). In particular, the stress-strain curves of models that differ only in

267 the location of initial seed points, which determine the random packing of particles, match along

$268>90 \%$ of their length preceding macroscopic failure, have comparable means and magnitudes of

269 fluctuations after failure, and produce similar failure stresses (Fig. S3).

270 The numerical models employed here benefit from several years of software development

271 and benchmark calibration (e.g., Weatherley et al., 2010; Schopfer et al., 2009; Mair and Abe,

272 2009). Consequently, we selected several model parameters, such as the ratio of the minimum to

273 maximum particle radii $(0.1 / 1.0)$, using results from previous calibrations that produce model

274 behaviors that closely match the behavior of linear elastic brittle materials.

\section{3.4. Internal friction derivation}

276 To constrain the macroscopic internal friction, $\mu_{0}$, of each simulated layered sedimentary

277 rock, we execute triaxial compression tests at increasing confining stress, $\sigma_{2}=\sigma_{3}$. We test $\sigma_{2}$ 
278 from 0-50 MPa in models with differing layer orientations (from horizontal to vertical layers),

279 differing layer interface roughness $(0.2 \mathrm{~mm}$ or $0.5 \mathrm{~mm}$ overlap) and differing total layer interface

280 area (cube or block models), requiring more than 256 unique model runs (Table S.2). In order to

281 compare the geomechanical responses of layered rock to more homogeneous rocks that do not

282 contain layers, and so assess when the impact of layers exerts a significant control on failure

283 behavior, we repeat this analysis on homogeneous block models with no layers.

284 At each applied $\sigma_{2}$, we find the peak failure stress, $\sigma_{\mathrm{F}}$. Rearranging the Coulomb shear failure

285 criterion in terms of the principal stresses and uniaxial compressive strength, $\mathrm{S}_{0}$, provides a

286 relationship for the internal friction angle, $\varphi$ (e.g., Jaeger et al., 2007):

287

$$
\sigma_{F}=S_{0}+\sigma_{2} \tan ^{2}\left(\frac{\pi}{2}+\frac{\varphi}{2}\right) .
$$

Eq. 2

288 We find the slope of the linear regression through $\sigma_{2}$ and $\sigma_{\mathrm{F}}$ of the models to calculate $\varphi$. We 289 report internal friction as the friction coefficient, $\mu_{0}=\tan \varphi$.

\section{4. Results}

291 First, we describe characteristic loading curves produced by the simulated rock (Fig. 3). We 292 analyze the distribution of $\sigma_{\mathrm{F}}$ and $\mu_{0}$ with respect to the applied confining stress, $\sigma_{2}$, and layer 293 orientation, $\theta$, in models with block and cube geometries. We show how strain localizes as the

294 models deform by presenting spatial distributions of particle velocities and nucleating fractures, 295 and the difference in the percentage of fractures that form along the preexisting weaknesses and 296 within the host matrix.

\section{4.1. Stress-strain relationships}

298 Typical loading curves from the numerical simulations share several characteristics with 299 laboratory experiments (Fig. 3). Consistent with triaxial compression experiments, we observe 1) 300 increasing failure stress, $\sigma_{\mathrm{F}}$, with increasing $\sigma_{2}$ (e.g., Mogi et al., 1967), 2) broadening of the 
301 loading curve with approaching macroscopic failure with increasing $\sigma_{2}$ (e.g., Niandou et al.,

302 1997), 3) decreasing stress drop with increasing $\sigma_{2}$ (e.g., Klein et al., 2001), and 4) decreasing

303 slope of the failure envelope with increasing $\sigma_{2}$ (e.g., Byerlee, 1978) (Fig. 3). The decreasing

304 slope of the failure envelope with increasing $\sigma_{2}$ may arise from the initial compaction of the

305 model preceding axial displacement loading, as the initial porosity is $24-25 \%$. These models do

306 not produce a clear transition from brittle to ductile behavior up to $50 \mathrm{MPa}$ confining stress. This

307 transition could occur at higher confining stresses, but as this study focuses on brittle failure, we

308 do not assess this possibility. This transition likely depends on the ratio of the normal and shear

309 stiffness of the bonds in DEM simulations.

310 Calculating the linear best-fit through the failure envelope of principal stresses $\left(\sigma_{\mathrm{F}}\right.$ and $\left.\sigma_{2}\right)$

311 produces values of $\mathrm{R}^{2}>0.95$ for all of the models, lending confidence to the $\mu_{0}$ estimates that we

312 derive from the linear regression through the full range of $\sigma_{2}$. This linearity, and resulting high

$313 \mathrm{R}^{2}$ values are consistent with the $\mathrm{R}^{2}$ values $(0.90-0.99)$ of linear regressions of experimental

314 failure envelopes of shale produced in direct shear tests (Heng et al., 2015). However, because

315 previous work (e.g., Byerlee, 1978) as well as our data indicates that the slope of the failure

316 envelope decreases at higher $\sigma_{2}$, we calculate $\mu_{0}$ using linear regressions through the failure

317 envelope for the subsets $\sigma_{2}=0-20 \mathrm{MPa}$, and $\sigma_{2}=20-50 \mathrm{MPa}$, as well as $\sigma_{2}=0-50 \mathrm{MPa}$ (Figs. S.4-

318 S.5).

319 The curvature of the failure envelope from 0-20 MPa, in particular, suggests that estimates of

320 internal friction using data from this range will underestimate friction at the lower end of this

321 range. However, the relatively high $\mathrm{R}^{2}$ values suggest that the difference between the true and

322 apparent internal friction will be small.

323 4.2. Impact of layer orientation on macroscopic geomechanical behavior 
The applied confining stress, $\sigma_{2}$, layer orientation, $\theta$, and overlap distance control the peak

325 failure stress, $\sigma_{\mathrm{F}}$ (Fig. 4). Consistent with laboratory experimental studies (e.g., Donath, 1961;

326 Duveau et al., 1998), $\sigma_{\mathrm{F}}$ is lowest when $\theta$ is $60^{\circ}$, and maximized at $90^{\circ}$ and $0-15^{\circ}$ (Fig. 4). For

327 each applied $\sigma_{2}$, the range of $\sigma_{\mathrm{F}}$ as a function of orientation is slightly greater in the block models

328 than the cube models (Fig. 4). This result arises because the block models have higher layer

329 interface area than the cube models. Varying the orientation of the layers therefore has a greater

330 impact on $\sigma_{\mathrm{F}}$ in the block models because they have larger ratios of the number of weak bonds to

331 strong bonds than the cube models. The range of $\sigma_{\mathrm{F}}$ is larger in models with smoother layer

332 interfaces ( $0.2 \mathrm{~mm}$ overlap) than in models with rougher layer interfaces. We expect that the

333 influence of layer orientation on failure will be magnified when the layer interfaces are smoother

334 compared to when they are rougher. When the layer interfaces are rougher, they function more

335 similarly to the host matrix rather than mechanical weaknesses. Notably, the $\sigma_{\mathrm{F}}$ for each $\sigma_{2}$ of

336 models with horizontal and vertical layers are almost identical to that of the homogeneous block

337 models.

338 Due to the steeping of the failure envelopes at lower $\sigma_{2}$ (Fig. 3), we determine $\mu_{0}$ using the

339 full $\sigma_{2}$ range (0-50 MPa), as well as lower (0-20 MPa) and higher (20-50 MPa) ranges (Fig. S4-

340 S5). As expected, $\mu_{0}$ calculated from the lower $\sigma_{2}$ range (red in Fig. 5) is larger than the upper $\sigma_{2}$

341 range (blue in Fig. 5), while the estimates derived from the full range sit between those extremes

342 (Fig. S4-S5, black in Fig. 5). In the block models, the distribution of $\sigma_{\mathrm{F}}$ produces similar values

343 of $\mu_{0}$ to cube models, with minima at $\theta=60^{\circ}$, i.e., at $30^{\circ}$ with respect to $\sigma_{1}$ (Fig. 5 ). The range of

$344 \mu_{0}$ is larger in the model with smooth than rough layer interfaces (Fig. 5).

345 In general, the homogeneous models produce estimates of $\mu_{0}$ that closely match estimates 346 from models with horizontal and vertical layers (Fig. 5). At confining stresses $<20 \mathrm{MPa}$, the 
347 presence of layers reduces $\mu_{0}$ from that of more homogeneous rock only when $\theta=30^{\circ}-60^{\circ}$. At

348 higher confining stresses, the layers produce $\mu_{0}$ that differs from the homogeneous models when $349 \theta=0^{\circ}-60^{\circ}$.

350 The cube models display a smaller range of $\mu_{0}$ values than in the block models (Fig. 5), 351 consistent with trend in $\sigma_{\mathrm{F}}$ described above, and can similarly be attributed to the reduced 352 interface area in the cube models relative to the block models. Some trends in the distribution of $353 \mu_{0}$ with respect to $\theta$ are similar between the block and cube models, including the maxima at $75^{\circ}$, 354 and minima at $60^{\circ}$. However, whereas the $\mu_{0}$ minimum occurs only at $60^{\circ}$ in the block models, 355 and in the cube models with smooth interfaces ( $0.2 \mathrm{~mm}$ overlap); in the rougher cube models, $\mu_{0}$ 356 is minimized when $\theta$ is $0^{\circ}, 30^{\circ}$ and $60^{\circ}$ (Fig. 5).

357 To investigate whether the anomalously high $\mu_{0}$ for models with $\theta=75^{\circ}$ arises from numerical 358 artifacts, we calculate $\mu_{0}$ for block models oriented at $\theta=70^{\circ}$ and $\theta=80^{\circ}$ because these angles 359 bracket $\theta=75^{\circ}$, for both smooth ( $0.2 \mathrm{~mm}$ overlap) and rough ( $0.5 \mathrm{~mm}$ overlap) interfaces. We 360 find that the resulting $\mu_{0}$ estimates when $\theta=70^{\circ}$ and $\theta=80^{\circ}$ similarly bracket this value at $\theta=75^{\circ}$ 361 (Fig. S6). This distribution of $\mu_{0}$ suggests that the high $\mu_{0}$ at $\theta=75^{\circ}$ is not an artifact of the model 362 set up, but reflects the magnified impact of $\sigma_{2}$ on $\sigma_{\mathrm{F}}$ at lower $\sigma_{2}$ compare to that impact at higher $363 \sigma_{2}$ for these steeper orientations $\left(70-90^{\circ}\right)$. This heightened sensitivity of $\sigma_{\mathrm{F}}$ to $\sigma_{2}$ produces steeper 364 slopes in the failure envelopes over the full $\sigma_{2}$ range, and particularly from $\sigma_{2}=0-20 \mathrm{MPa}$, which 365 produces higher $\mu_{0}$ when $\theta=70-90^{\circ}$ (Fig. S6). As the layer orientation steepens from $60^{\circ}$ to $80^{\circ}$, $366 \sigma_{F}$ at each tested confining stress gradually increases (Fig. S6). However, when the layer 367 orientation steepens from $80^{\circ}$ to $90^{\circ}, \sigma_{F}$ at confining stresses $<20 \mathrm{MPa}$ increase by larger 368 magnitudes than those increases from $60^{\circ}$ to $80^{\circ}$. This sudden jump suggests that the results at $36990^{\circ}$ are more anomalous than those at $75^{\circ}$. In other words, if the $\sigma_{F}$ under lower confining 
stresses $(<20 \mathrm{MPa})$ at $90^{\circ}$ more closely matched those at $80^{\circ}$, then the trend in internal friction

371 would more closely match expectations: gradually increasing from $60^{\circ}$ to $90^{\circ}$. The applied

372 microparameter tensile strength may have produced this anomalous result. Lower tensile strength

373 would promote failure along planes orientation parallel to $\sigma_{1}$ (at $90^{\circ}$ ), producing lower $\sigma_{F}$ at

374 lower confining stresses and thus higher internal friction coefficients.

375 Similarly, Cho et al. (2012) found overlapping values of uniaxial compressive strength when $376 \theta=75^{\circ}$ and $\theta=90^{\circ}$ in laboratory tests on layered shale. The difference in layer orientation did not 377 appear to influence the orientation of system-spanning fractures in these experiments, with both 378 tend to trend parallel to $\sigma_{1}$. In our numerical simulations, the steepening of layers by $15^{\circ}$ toward $379 \sigma_{1}$ do not significantly increase the failure strength at higher confining stresses $(>30 \mathrm{MPa})$, but 380 do lead to larger increases in strength at lower confining stresses (<30 MPa). This difference in 381 behavior likely arises from the ability of the cores to fail via axial splitting along fractures 382 trending vertical to $\sigma_{1}$ (promoted under lower confining stresses), or along more-obliquely 383 oriented fractures (promoted under higher confining stresses).

384 Comparing the magnitudes of $\mu_{0}$ produced in models with each of the assessed parameters 385 held constant reveals the relative impact of each individual parameter (Fig. 6, Fig. S7). Models 386 with the largest interface area (blocks) and the smoothest layers $(0.2 \mathrm{~mm})$ produce the largest 387 changes in $\mu_{0}$ (Fig. 6A). In general, smoother interfaces promote slightly lower values of internal 388 friction than rough interfaces, with the largest variations when layers are oriented at $\theta=45^{\circ}$ and $38960^{\circ}$ (Fig. 6B, D). Internal friction values are similar for cubes (with lower interface area) and 390 blocks (with higher interface area) except for layers oriented at $\theta=60^{\circ}$ and $75^{\circ}$, where $\mu_{0}$ for the 391 blocks and cubes diverge the most (Fig. 6C, D). Varying the layer orientation produces the 392 largest $\Delta \mu_{0}$ compared to varying the layer interface roughness or model shape (Fig. 6A, D). 
393 Varying the shape of the model (cube or block) produces the smallest changes in $\mu_{0}$ relative to

394 the layer orientation and layer roughness (Fig. 6C, D).

395 The distribution of elastic modulus relative to the layer orientation produced in these

396 numerical models are consistent with laboratory experiments on layered shale (Cho et al., 2012).

397 In both these numerical models (Fig. S8) and experiments, the effective elastic modulus

398 increases as the orientation of layers become increasingly parallel to $\sigma_{1}$.

\subsection{Linking microstructural deformation to macroscopic behavior}

$400 \quad$ To understand how the preexisting weaknesses influence the location and organization of 401 microscopic failure, we show the spatial distribution of particle velocities (Fig. 7) and nucleating 402 fractures (Fig. 8) as the simulated rock begins to fail. In these models, the bonds that break in 403 each time step are analogous to nucleating or growing fractures in natural rock, and so we refer 404 to them as nucleating fractures. Similarly, just as microfractures coalesce to form macroscopic 405 faults in natural rocks, many bonds may break in clusters and thus form through-going faults. 406 Particle velocities reveal structural reorganization within the rock at a micro- and mesoscopic 407 scale. We focus on block models with $0.2 \mathrm{~mm}$ overlap because these models produce the largest 408 variation in geomechanical behavior as the layer orientation changes. We report results from two 409 representative confining stresses $(0 \mathrm{MPa}, 10 \mathrm{MPa})$ in models with end-member layer orientations $410\left(\theta=0^{\circ}, 90^{\circ}\right)$, and the orientation that produces the lowest $\sigma_{\mathrm{F}}$ and $\mu_{0}$ in the block models, $\theta=60^{\circ}$.

411 In models with horizontal layers $\left(\theta=0^{\circ}\right)$, and no confining stress, early in the experiment, the 412 spatial distributions of particle velocities (Fig. 7A, Animation S1) and fractures (Fig. 8A, 413 Animation S1) indicate that the weaker layer interfaces accommodate more deformation than 414 within the layers, and later deformation spreads to the host matrix. Initially, the upper layer 415 concentrates deformation, and then as the lower layer begins to break, bond breakage penetrates 
416 this lower layer in a diffuse volume that is bounded by inclined planes (Fig. 8A). At higher

417 confining stress (10 MPa), the spatial distribution of particle velocities (Fig. 7D, Animation S2)

418 and fractures (Fig. 8D, Animation S2) reveal that deformation is more equally distributed

419 throughout the host matrix. Deformation appears to be localized onto the weak bonds between 420 layers only early in the experiment, whereas after 0.003 of axial strain, bonds break throughout

421 the model apparently irrespective of the location of the weaknesses (Fig. 8D). After this axial 422 strain, the fractures align into inclined pseudo-planar volumes. The confining stress appears to

423 suppress the influence of the weak structure. The deformation patterns produced under no and 10

424 MPa confining stress resemble macroscopic failure produced by the propagation of a through425 going fault.

426 In models with layers oriented at $\theta=60^{\circ}$ under zero or no confining stress, the spatial 427 distributions of particle velocities (Fig. 7B, Animation S3) and fractures (Fig. 8B, Animation S3) 428 indicate that a subset of the layer interfaces preferentially accommodate slip throughout much of 429 the experiment. The few dominantly active interfaces are separated by several intermediate 430 passive layers, causing the rock to fail as if it had fewer and thicker layers. However, velocity 431 gradients within the emergent layers indicate that slip along the interfaces does not accommodate 432 all of the deformation and some damage is located within the host (Fig. 7B). Early in the 433 simulation, fractures form along all of the preexisting layer interfaces, whereas with continued 434 strain, slip localizes onto only a few of the available slip surfaces (Fig. 8B, Animation S3). The 435 observed strain localization onto a portion of the available surfaces suggests that the thickness of 436 preexisting layers (i.e., the total layer interface area) does not exert a primary control on strain 437 localization and the resulting geomechanical behavior when $\theta=60^{\circ}$. Similarly, at higher 438 confining stress (10 MPa), the spatial distributions of particle velocities (Fig. 7E, Animation S4) 
and fractures (Fig. 8E, Animation S4) indicate that early in the experiment, weak bonds between

440 layers preferentially localize deformation, but after 0.00103 axial strain, deformation localizes

441 onto only a few interfaces.

442 In models with vertical layers $\left(\theta=90^{\circ}\right)$ under no confining stress, the spatial distributions of 443 particle velocities (Fig. 7C, Animation S5) and fractures (Fig. 8C, Animation S5) reveal that the

444 vertical layers facilitate axial splitting. This macroscopic failure behavior arises from tensile

445 failure along the layer interfaces. In addition, similar to the models with horizontal layers,

446 inclined planes of nucleating fractures penetrate within layers later in deformation. Differences

447 in particle velocities across layer interfaces indicate some slip across layer interfaces, but at

448 lower magnitudes than observed in models with layers dipping at $60^{\circ}$ (Fig. 7B). Gradients in the

449 velocity fields within individual layers highlight that slip and opening along the interfaces and

450 breakage in the top portion of the block did not accommodate all of the deformation within the

451 rock. At higher confining stress $(10 \mathrm{MPa})$, the spatial distributions of particle velocities (Fig. 7F,

452 Animation S6) and fractures (Fig. 8F, Animation S6) indicate that the vertical layers do not

453 appear to concentrate deformation along their interfaces as the rock fails, unlike the zero

454 confining stress case. Instead, deformation gradually localizes onto an oblique fracture oriented

455 at approximately $30^{\circ}$ to $\sigma_{1}$ that accommodates both shear and tensile failure. The impact of the

456 vertical weak interfaces, similarly to horizontal layers (Fig. 7D, Fig. 8D, Animation S2), appears

457 to be suppressed by confining stress, perhaps because the axial splitting mechanism (Fig. 7C,

458 Animation S5) can no longer operate as effectively under the applied confining stress.

459 In homogeneous models that lack preexisting layers, the spatial distributions of particle

460 velocities and fractures (Animations S7-S8) appear similar to those observed in models with

461 horizontal layers, consistent with the similar failure stresses and internal friction of those sets of 
462 models. In particular, under no confining stress, the rock begins to fail through the development

463 of a broad zone of fractures at a top corner of the model that spreads into the block along a plane

464 inclined at around $30^{\circ}$ to $\sigma_{1}$.

465 4.4. Preferential strain localization along layer interfaces

466 To quantify the degree and rate of strain localization onto preexisting weaknesses relative to

467 the host rock, we track the number of (weak) interface bonds that break in each model time step

468 versus (strong) host rock bonds (Fig. S9, Fig. 9). We calculate the percentage of stronger host

469 bonds (out of the total strong bonds), $\mathrm{B}_{\mathrm{s}}$, and the percentage of weak interface bonds (out of the

470 total weak bonds), $B_{w}$, that break (Fig. S9). We report the difference $B_{w}-B_{s}$, as parameter $\Delta B$.

471 This parameter reveals the extent to which localized fracture development occurs along the

472 preexisting weaknesses as opposed to within the host rock. A large value of $\Delta \mathrm{B}$ indicates damage

473 is preferentially localized along the weak interfaces whereas a smaller value indicates a reduced

474 impact of the weak interfaces. The evolution of $\Delta \mathrm{B}$ is presented as a function of the applied

475 strain (normalized by failure strain) for the full range of tested confining stresses and interface

476 orientations (Fig. 9). Trends are similar in block and cube models, as well as for rougher and

477 smoother layer interfaces (Fig. S9), so we focus on the block models with smoother layer

478 interfaces (Fig. 9).

479 Preceding failure in each model, $\Delta \mathrm{B}$ is positive because the percentage of weak bonds that

480 break are always higher than the percentage of strong bonds (Fig. 9, Fig. S9). In each model,

481 there is an initial increase in $\Delta \mathrm{B}$ with applied strain, but in some cases $\Delta \mathrm{B}$ decreases as the rock

482 approaches failure. The layer orientation exerts a stronger influence on the evolution of $\Delta \mathrm{B}$ than

483 the $\sigma_{2}$ (Fig. 9). At each $\sigma_{2}$, the $\Delta \mathrm{B}$ evolution is relatively similar for constant $\theta$ (Fig. 9A-C). At

484 differing $\theta$, variations in $\sigma_{2}$ do not produce as significant changes in the overall $\Delta \mathrm{B}$ evolution, 
except at higher $\theta$ (Fig. 9D-F). At higher $\theta$, the differences are systematic, and produced by the

486 suppression of fractures opening in the direction of the applied confining stress. In particular, the

487 overall slope of the $\Delta \mathrm{B}$ curve decreases as confining stress increases when the layers are vertical.

488 The impact of $\sigma_{2}$ on the $\Delta \mathrm{B}$ evolution increases with increasing $\theta$ because the applied confining

489 stress prevents some opening along layer interfaces in models with vertical layers, and does not

490 prevent this opening in models with more shallowly dipping layers.

491 The $\Delta \mathrm{B}$ evolution reflects the impact of the microstructural reorganization and damage

492 observed in the spatial distribution of particle velocities (Fig. 7) and fractures (Fig. 8). At lower $\theta$

$493\left(0-15^{\circ}\right)$, the $\Delta \mathrm{B}$ increases, then flattens, and decreases as the rock approaches failure (Fig. 9A-

494 D). This decrease indicates that an increasing percentage of strong bonds relative to the

495 percentage of weak bonds are breaking in each time step. The spatial distribution of particle

496 velocities and fractures in the model with horizontal layers also indicate that a decreasing

497 number of bonds break along layer interfaces throughout the experiment (Fig. 7, Fig. 8).

498 When $\theta$ is $60^{\circ}$, and $\sigma_{\mathrm{F}}$ and $\mu_{0}$ are minimized, $\Delta \mathrm{B}$ and the rate of $\Delta \mathrm{B}$ continually increase

499 preceding macroscopic failure for each $\sigma_{2}$. The $\Delta \mathrm{B}$ evolution (Fig. 9), and spatial distribution of

500 particle velocities (Fig. 7) and nucleating fractures (Fig. 8) demonstrate that slip along the layer

501 interfaces preferentially accommodates the majority of deformation throughout the models with

$502 \theta=60^{\circ}$. Gradients in the particle velocity fields within individual layers and the presence of

503 fractures nucleating away from the weak interfaces demonstrate that damage in the host rock

504 accommodates a portion of the total strain, and the $\Delta \mathrm{B}$ evolution quantifies the degree of strain

505 localization onto the preexisting weaknesses.

506 To further assess the relative influence of layer orientation and confining stress on strain

507 localization, we calculate the anisotropy ratio $\mathrm{K}_{2}$ (Fig. 10). Following the approach of Lisjak et 
508 al. (2014), $\mathrm{K}_{2}$ is the ratio of the maximum to minimum differential stress at failure of

509 experiments with equal confining stress and differing layer orientation:

$$
K_{2}=\frac{\left(\sigma_{1}-\sigma_{2}\right)_{\max }}{\left(\sigma_{1}-\sigma_{2}\right)_{\min }} \quad \text { Eq. } 3
$$

511 High $\mathrm{K}_{2}$ indicates larger differences between the failure stresses of the weakest and strongest

512 models. Consistent with Lisjak et al. (2014), $\mathrm{K}_{2}$ decreases as confining stress increases, and

513 begins to plateau when $\sigma_{2}>20 \mathrm{MPa}$. Comparing the relationship between $\mathrm{K}_{2}$ and confining stress

514 for models with smoother and rougher layer interfaces and block- and cube-shapes indicates that

515 smoother layer interfaces and greater interface area produce higher anisotropic ratios. This result

516 arises because smoother layers and more area available for slip magnify the impact of the

517 preexisting weaknesses on strain localization and subsequent failure. In addition, our

518 comparisons of $\mathrm{K}_{2}$ indicate that layer interface roughness more strongly controls the evolving

519 anisotropy of macroscopic failure strength $\left(\mathrm{K}_{2}\right)$ at differing confining stresses than the model

520 shape, and hence interface area available for slip.

\section{5. Discussion}

\section{5.1. Influence of planar preexisting weaknesses on failure behavior}

524 Our results constrain the manner in which preexisting mechanical weaknesses in layered 525 rocks influence microscopic deformation that leads to macroscopic failure. Constraining the 526 impact of heterogeneities on internal friction is critical for the robust assessment of intact

527 material failure, and in the definition of safety factors when estimating the strength of

528 geomaterials. We show that lower confining stress, intermediately-dipping weaknesses with 529 respect to $\sigma_{1}$, smoother layer interfaces, and higher preexisting interface area available for slip 530 magnify the impact of microscopic weaknesses on the macroscopic geomechanical behavior 
531 (Fig. 11). Higher confining stress, more steeply or shallowly dipping weaknesses relative to $\sigma_{1}$,

532 rougher interfaces and less layer interface area available for slip reduce this impact. The relative

533 importance of each of these parameters on internal friction within the explored parameter space,

534 from most to least important is 1) confining stress, 2) weakness orientation relative to $\sigma_{1}, 3$ )

535 interface roughness, and 4) interface area (Fig. 11, Fig. 6).

536 Previous analyses reported trends similar to those observed here between individual

537 parameters and failure behavior, such as preexisting weakness orientation and uniaxial

538 compressive strength (e.g., Lisjak et al., 2014). Here and in previous studies, $\sigma_{\mathrm{F}}$ is minimized

539 when $\theta$ is $60^{\circ}$, and maximized at $90^{\circ}$ and $0-15^{\circ}$ (e.g., Donath, 1961; Duveau et al., 1998; Cho et

540 al., 2012). Similarly, experimental studies have found that the internal friction coefficient is

541 minimized at $60^{\circ}$ and maximized at $0^{\circ}$ and $90^{\circ}$ (e.g., Donath, 1961 ; Nova, 1980). Our numerical

542 distribution of strength relative to anisotropy orientation agrees better with experimental results

543 than previous numerical analyses of FEM/DEM-DFN models (Lisjak et al., 2014) and DEM

544 models (Dinç and Scholtès, 2018) that find minima in failure strength when preexisting

545 anisotropies are oriented $45^{\circ}$ from $\sigma_{1}$.

546 The present numerical contribution extends our understanding of the relative impact and

547 importance of each parameter on failure behavior as well as the interplay between these

548 parameters. Furthermore, our study directly examines the micromechanical processes that

549 produce the resulting macroscopic behavior, and so provides insights into microstructural

550 sources that produce particular macroscopic geomechanical behavior.

551 Such insights are generally not yet available experimentally without advanced techniques

552 such as digital volume correlation (DVC) analysis of in situ microtomograms. DVC analysis

553 provides local strain tensors within elastically and inelastically deforming material. Recent DVC 
554 analysis on experiments with laminated shale examines microscopic strain localization preceding

555 macroscopic failure, and links the observed local strain evolution to macroscopic failure

556 behavior (e.g., McBeck et al., 2018). These time series of local strain tensors demonstrate that

557 the lamination bedding plane orientation of the shale controls the evolving spatial distribution of

558 local normal strains prior to failure. Furthermore, associated numerical investigations indicate

559 that the localization of normal strains in turn controls the localization of shear strains. These

560 differences in the microscopic strain evolution produce differences in the macroscopic behavior:

561 the shale core fails at a higher differential stress when its laminations are set perpendicular to $\sigma_{1}$

562 than when its laminations are subparallel to $\sigma_{1}$. In contrast, the uniaxial compressive strength of

563 the present numerical models with layers set perpendicular to $\sigma_{1}$ are slightly lower than the

564 strength of models with layers parallel to $\sigma_{1}$. This difference may arise from the lack of

565 truncation factor used to specify the tensile strength of the bonds. In the present models,

566 extension of the Mohr-Coulomb criterion into the tensile regime using the bond internal friction

567 and bond cohesion determine the bond tensile strength. The application of a truncation factor

568 reduces the bond tensile strength from that predicted from the microparameter Mohr-Coulomb

569 criterion into the tensile regime. The measured tensile strength of rocks is often lower than that

570 predicted from the Coulomb criterion. Although microparameter values are not expected to equal

571 macroscopic rock values, if a truncation threshold was employed, models with layers parallel to

$572 \sigma_{1}$ may have lower failure strengths than models with layers perpendicular to $\sigma_{1}$.

573 Models with the smoothest layer interfaces and greatest interface area produce the largest

574 ranges in $\sigma_{\mathrm{F}}$ and $\mu_{0}$. In these models, the simulated rock is geomechanically the weakest when

575 the weaknesses are oriented at $\theta=60^{\circ}\left(\sigma_{\mathrm{F}}=40 \mathrm{MPa}\right.$ at zero confining stress), and strongest when

576 the layers are parallel or perpendicular to $\sigma_{1}\left(\sigma_{\mathrm{F}}=120 \mathrm{MPa}\right.$ at zero confining stress $)$. Similarly, the 
577 rock has the lowest $\mu_{0}$ for layers at $\theta=60^{\circ}(0.55)$, and highest $\mu_{0}$ for layers oriented near parallel

578 and perpendicular to $\sigma_{1}(0.64-0.68)$. Estimates of $\mu_{0}$ from the homogeneous models that lack

579 layers exceed estimates of $\mu_{0}$ from the layered models except when $\theta=70-90^{\circ}$. Consequently, the

580 impact of preexisting weaknesses on $\mu_{0}$ and the resulting likelihood of failure is particularly

581 important when $\theta=0-60^{\circ}$, and less important when layers are sub-parallel to $\sigma_{1}$. The difference

582 between the $\mu_{0}$ calculated for the lower (0-20 MPa) and higher (20-50 MPa) $\sigma_{2}$ ranges is $0.15-$

5830.2 , suggesting that decreasing $\sigma_{2}$ by $20 \mathrm{MPa}$ can increase the effective $\mu_{0}$ by up to 0.2 . Varying

584 the orientation of the weaknesses may change $\sigma_{\mathrm{F}}$ by $66 \%$, and $\mu_{0}$ by $20 \%$ respectively.

585 The spatial distributions of fractures and particle velocities reveal that at low confining stress

586 (0 MPa), the preexisting weaknesses impact both strain localization and macroscopic failure for

587 all of the tested orientations (Fig. 7, 8). However, at higher confining stress (10 MPa), strain

588 preferentially localizes onto the layer interfaces only when the layers are dipping at intermediate

589 angles $\left(\theta=60^{\circ}\right)$. When the layers are more shallowly or steeply dipping $\left(\theta=0^{\circ}\right.$ and $\left.90^{\circ}\right)$ strain

590 effectively ignores the weak layers (Fig. 7, 8). The exploitation of the weaker layer contacts with

591 intermediately dipping angles throughout deformation, and throughout the range of tested

592 confining stresses, produces the lower failure stress and lower internal friction of these simulated

593 layered rocks. Similarly, layered rocks with preexisting weaknesses at higher and lower

594 orientations, i.e. subparallel or subperpendicular to $\sigma_{1}$, have higher failure stresses and higher

595 internal friction because the microscopic deformation does not exploit the preexisting

596 weaknesses as much as rocks with more intermediately dipping layers. This lack of exploitation

597 causes the strength of the bulk matrix, rather than the strength of the preexisting weaknesses, to

598 control the macroscopic strength in these rocks. 
Early in the experiment when $\theta=60^{\circ}$, slip is nearly equally distributed amongst the

600 preexisting layer interfaces, but in subsequent stages, slip localizes onto only one or two layer

601 interfaces. This evolution produces emergent layers that are thicker than the preexisting layers.

602 This behavior suggests that when the layers are oriented favorably for slip $\left(\theta=45^{\circ}-60^{\circ}\right)$, the

603 initial width of layers may influence the onset of failure, but following the initial stage, the layer

604 width does not significantly impact failure behavior and the macroscopic geomechanical

605 properties.

606 Post-failure scanning electron microscope images of Tournemire shale provide evidence of

607 similar interactions between preexisting weaknesses and microfractures (Bonnelye et al., 2017a).

608 In these experiments, fractures tend to develop parallel to bedding planes when the orientation of

609 preexisting weakness are intermediate to $\sigma_{1}$. When this orientation is parallel or perpendicular to

$610 \sigma_{1}$, fractures typically cross cut bedding planes. This varying degree to which fractures exploit

611 preexisting weaknesses also arises in our numerical models. These experimental results provide

612 important validation of the microstructural deformation observed in our numerical work.

613 Furthermore, our numerical models and experimental work (Bonnelye et al., 2017a; Holt et al.,

614 2015) demonstrate that both confining stress and layer orientation impact the degree to which

615 fractures exploit weaknesses.

616 In this numerical work, we quantify the relative impact of confining stress and layer

617 orientation on failure behavior using $\Delta \mathrm{B}$ (Fig. 9) and $\mathrm{K}_{2}$ (Fig. 10). In addition, our models

618 highlight the evolution of the degree of exploitation of preexisting weaknesses preceding failure,

619 and not only as post-failure snapshots retrieved from experiments (e.g., Bonnelye et al., 2017a),

620 or inferred from elastic wave measurements (e.g., Bonnelye et al., 2017b). In particular, our

621 results enable quantitative tracking of the rate and degree of strain localization onto preexisting 
622 weaknesses relative to the host rock, $\Delta \mathrm{B}$, throughout loading preceding failure (Fig. 9). This

623 quantification demonstrates that the orientation of preexisting weaknesses more strongly controls

624 the degree and rate of strain localization than the imposed confining stress (Fig. 9). Furthermore,

625 the degree and rate of strain localization continually increase in models with the lowest failure

626 strengths (layers oriented $30^{\circ}$ from $\sigma_{1}$ ), but plateau in models with higher failure strengths (layers

627 oriented $90^{\circ}$ from $\sigma_{1}$ ). However, in models with higher failure strengths and layers oriented

628 parallel to $\sigma_{1}$, such a plateau in $\Delta \mathrm{B}$ only occurs under higher confining stresses ( $>40 \mathrm{MPa}$ ), and

629 an acceleration in $\Delta \mathrm{B}$ occurs under lower confining stress. Consequently, the varying evolutions

630 of $\Delta \mathrm{B}$ demonstrate how the evolving spatial distribution of microfractures and their localization

631 onto preexisting weakness planes control macroscopic failure behavior. Tracking the anisotropic

632 ratio $\mathrm{K}_{2}$ demonstrates that the roughness of layer interfaces exert greater control on differing

633 failure behavior than the total layer interface area available for slip (Fig. 10).

\section{5.2. Micromechanical models of anisotropic rock failure}

635 Previous experimental studies have evaluated the impact of preexisting weaknesses on

636 geomechanical behavior using laboratory data. For example, Duveau et al. (1998) compare the

637 predictions of nine failure criteria to data from laboratory triaxial compression experiments on

638 Angers schist. They conclude that discontinuous weakness planes models are particularly apt to

639 capture the failure of anisotropic material because they are based on the two dominant failure

640 mechanisms within anisotropic rock: failure along planes of weakness and failure elsewhere

641 within the host rock. Of the discontinuous weakness planes models, our approach to evaluating

642 the impact of weaknesses on failure is most similar to Jaeger's single plane of weakness theory

643 (Jaeger, 1960). This formulation uses two failure envelopes following the Mohr-Coulomb failure

644 criterion constructed from two sets of mechanical properties that represent failure along a plane 
645 of weakness or failure within the host rock. The ranges in macroscopic geomechanical properties

646 produced by deformation of our simulated layered rock enable construction of these envelopes

647 with end-members identified in the simulations. Using the values from block models with

648 smoother layer interfaces when $\theta=60^{\circ}\left(\sigma_{\mathrm{F}}=40 \mathrm{MPa}\right.$ under no confining stress, $\left.\mu_{0}=0.55\right)$ and

$649 \theta=90^{\circ}\left(\sigma_{\mathrm{F}}=120 \mathrm{MPa}\right.$ under no confining stress, $\left.\mu_{0}=0.68\right)$, at $7 \mathrm{~km}$ depth, a rotation of $\sigma_{1}$ from $0^{\circ}$

650 to $30^{\circ}$ from the layer orientation (or a $30^{\circ}$ shift in the orientation of layering from $\sigma_{1}$ ) is predicted

651 to reduce the shear stress required for failure by $104 \mathrm{MPa}$. Typical coseismic stress drops range

652 between 1-10 MPa (e.g., Smith and Sandwell, 2003), and stress changes of 0.1 MPa may be

653 sufficient to trigger earthquakes (King et al., 1994), indicating that a decrease in $104 \mathrm{MPa}$ is

654 significant in the context of failure of preexisting faults in the crust. When considering the failure

655 of intact sedimentary rock, a decrease in $104 \mathrm{MPa}$ is significant because it approaches estimates

656 of the uniaxial compressive strength of sedimentary rock. These results indicate that varying the

657 orientation of weaknesses or $\sigma_{1}$ can cause intact layered rock to behave as if it hosted a

658 preexisting fault with no cohesion. Major earthquakes can rotate principal stress axes in tectonic

659 settings (e.g., Hardebeck \& Okada, 2018), and so may trigger this shift in the effective strength

660 of the crust.

661 Recent analyses of series of microtomograms captured in situ throughout triaxial

662 compression indicate that the failure of relatively isotropic and low-porosity rock (quartz-

663 monzonite) behaves as a dynamical critical phase transition (Renard et al., 2018). Future

664 analyses should target whether the failure of more mechanically anisotropic rock behaves

665 critically, and/or is adequately predicted by other failure models, such as the sliding wing-crack

666 model (Ashby \& Sammis, 1990), the Drucker-Prager failure criterion (Drucker \& Prager, 1952),

667 a modified Mohr-Coulomb criterion that considers intermediate principal stresses and non- 
668 linearity (Singh and Singh, 2011), or a discontinuous weakness planes model, such as Jaeger's

669 criterion (Jaeger, 1960).

670 6. Conclusions

671 Constraining the impact of mechanical weaknesses on strain localization and macroscopic

672 shear failure is critical for predicting the failure of buildings, tunnels, intact rocks, or preexisting

673 faults. Our numerical triaxial compression experiments on layered sedimentary rock produce

674 loading curves that broaden with increasing $\sigma_{2}$, stress drops that increase with increasing $\sigma_{2}$, and

675 failure envelopes that decrease in slope at higher $\sigma_{2}$. The distributions of $\sigma_{\mathrm{F}}$ and $\mu_{0}$ relative to the

676 layer orientation share characteristics observed in laboratory experiments (e.g., Donath, 1961),

677 with minimum $\sigma_{\mathrm{F}}$ and $\mu_{0}$ near $30^{\circ}$, and maxima near $0^{\circ}$ and $90^{\circ}$ from $\sigma_{1}$. A $30^{\circ}$ rotation in layer

678 orientation (or $\sigma_{1}$ ) produces a $66 \%$ and $20 \%$ difference in $\sigma_{\mathrm{F}}$ and $\mu_{0}$, respectively. Within the

679 upper seismogenic zone $(7 \mathrm{~km})$ in a sedimentary basin, this difference in mechanical properties

680 may decrease the shear stress required for Coulomb shear failure by $100 \mathrm{MPa}$. The degree of

681 strain localization from along preexisting weaknesses, rather than the host rock, continually

682 accelerates in rocks with the lowest failure strengths, but plateaus in rocks with higher failure

683 strengths, indicating that the rate of microscopic strain localization controls macroscopic

684 strength. Of the parameter space explored here, including layer orientation, layer interface

685 roughness, and total layer interface area, varying the layer orientation produces the largest

686 change in $\mu_{0}$, and varying total layer interface area produces the smallest change in $\mu_{0}$.

\section{Acknowledgements}

689 This study received funding from the Norwegian Research Council (project HADES, grant

690 250661) and computational resources from UNINETT Sigma2 AS, national infrastructure for 
691 computational science in Norway (project NN4557K). We thank Dion Weatherley and Steffen

692 Abe for fruitful discussions. We thank Editor Agard and two anonymous reviewers for

693 constructive comments that improved the manuscript. 


\section{References}

695 Abe, S., and Mair, K. (2005), Grain fracture in 3D numerical simulations of granular shear, Geophys. Res. Lett., 32, L05305, doi:10.1029/2004GL022123.

Abe, S., and Mair, K. (2009), Effects of gouge fragment shape on fault friction: New 3D modelling results. Geophysical Research Letters, 36(23), doi: 10.1029/2009GL040684.

Abe, S., Place, D., and Mora, P. (2003), A parallel implementation of the lattice solid model for the simulation of rock mechanics and earthquake dynamics, Pure. Appl. Geophys., 161, 2265-2277, doi: 10.1007/s00024-004-2562-x.

Belheine, N., Plassiard, J. P., Donzé, F. V., Darve, F., \& Seridi, A. (2009). Numerical simulation of drained triaxial test using 3D discrete element modeling. Computers and Geotechnics, $36(1-2), 320-331$.

Bonnelye, A., Schubnel, A., David, C., Henry, P., Guglielmi, Y., Gout, C., Fauchille, A. \& Dick, P. (2017a). Strength anisotropy of shales deformed under uppermost crustal conditions. Journal of Geophysical Research: Solid Earth, 122(1), 110-129.

Bonnelye, A., A. Schubnel, C. David, P. Henry, Y. Guglielmi, C. Gout, A.-L. Fauchille, and P.

736 Byerlee, J. (1978). Friction of rocks. Pure and applied Geophysics, 116(4), 615-626.

737 Cundall, P. A., and O. Strack (1979), A discrete numerical model for granular assemblies, 738 Geotechnique, 29, 47-65.

739 Cho, J. W., Kim, H., Jeon, S., \& Min, K. B. (2012). Deformation and strength anisotropy of Asan gneiss, Boryeong shale, and Yeoncheon schist. International Journal of Rock

742 Courant, R., Friedrichs, K., \& Lewy, H. (1928). On the partial difference equations of mathematical physics. Mathematische Annalen, 100.

Dinç, Ö., \& Scholtès, L. (2018). Discrete Analysis of Damage and Shear Banding in Argillaceous Rocks. Rock Mechanics and Rock Engineering, 51(5), 1521-1538. 
Donath, F. A. (1964). Strength variation and deformational behavior in anisotropic rock. State of Stress in the Earth's Crust, 281-298, edited by W. R. Judd, pp. 281-297, Elsevier Sci., New York.

Donath, F. A. (1961). Experimental study of shear failure in anisotropic rocks. Geological Society of America Bulletin, 72(6), 985-989.

Drucker, D. C., \& Prager, W. (1952). Soil mechanics and plastic analysis or limit design. Quarterly of Applied Mathematics, 10(2), 157-165.

Duveau, G., Shao., J., \& Henry, J. (1998). Assessment of some failure criteria for strongly anisotropic geomaterials. Mechanics of Cohesive-frictional Materials, 3(1), 1-26.

Fjær, E., \& Nes, O. M. (2014). The impact of heterogeneity on the anisotropic strength of an outcrop shale. Rock mechanics and rock engineering, 47(5), 1603-1611, doi: 10.1007/s00603-014-0598-5.

Gale, J. F., Laubach, S. E., Olson, J. E., Eichhubl, P., \& Fall, A. (2014). Natural fractures in shale: A review and new observations. AAPG bulletin, 98(11), 2165-2216, doi: $10.1306 / 08121413151$.

Hardebeck, J. L., \& Okada, T. (2018). Temporal Stress Changes Caused by Earthquakes: A Review. Journal of Geophysical Research: Solid Earth, 123, 13501365. doi:10.1002/2017JB014617.

Hazzard, J. F., and Mair, K. (2003). The importance of the third dimension in granular shear, Geophys. Res. Lett., 30(13), 1708, doi:10.1029/2003GL017534.

Hentz, S., Daudeville, L., \& Donzé, F. V. (2004). Identification and validation of a discrete element model for concrete. Journal of engineering mechanics, 130(6), 709-719.

Heng, S., Guo, Y., Yang, C., Daemen, J. J., \& Li, Z. (2015). Experimental and theoretical study of the anisotropic properties of shale. International Journal of Rock Mechanics and Mining Sciences, 74, 58-68.

Holt, R. M., Fjær, E., Stenebråten, J. F., \& Nes, O. M. (2015). Brittleness of shales: relevance to borehole collapse and hydraulic fracturing. Journal of Petroleum Science and Engineering, 131, 200-209. 
774 Jaeger, J.C. (1960). Shear failure of anisotropic rocks. Geological Magazine, 97(1), 65-72.

775 Jaeger J., Cook, N., \& Zimmerman, R. (2007). Fundamentals of rock mechanics. $4^{\text {th }}$ ed. Oxford: $776 \quad$ Blackwell.

777 King, G. C. P., Stein, R. and Lin, J. (1994). Static stress changes and the triggering of 778 earthquakes, Bull. Seismol. Soc. Am., 84, 935- 953.

779 Klein, E., Baud, P., Reuschlé, T., \& Wong, T. F. (2001). Mechanical behaviour and failure mode of Bentheim sandstone under triaxial compression. Physics and Chemistry of the Earth, Part A: Solid Earth and Geodesy, 26(1-2), 21-25.

Lisjak, A., Tatone, B. S., Grasselli, G., \& Vietor, T. (2014). Numerical modelling of the anisotropic mechanical behaviour of Opalinus clay at the laboratory-scale using FEM/DEM. Rock mechanics and rock engineering, 47(1), 187-206.

Mair, K., and Abe, S. (2008). 3D numerical simulations of fault gouge evolution during shear: Grain size reduction and strain localization, Earth Planet. Sci. Lett., 274, 72-81, doi:10.1016/j.eps1.2008.07.010.

Mair, K., \& Hazzard, J. F. (2007). Nature of stress accommodation in sheared granular material: Insights from 3D numerical modeling. Earth and Planetary Science Letters, 259(3-4), 469-485.

Mair, K., Main, I., \& Elphick, S. (2000). Sequential growth of deformation bands in the laboratory. Journal of Structural Geology, 22(1), 25-42.

McBeck, J., Kobchenko, M., Hall, S., Tudisco, E., Cordonnier, B., Meakin, P., \& Renard, F. Investigating the onset of strain localization within anisotropic shale using digital volume correlation of time-resolved X-ray microtomography images. Journal of Geophysical Research: Solid Earth. https://doi.org/10.1029/2018JB015676.

Mogi, K. (1967). Effect of the intermediate principal stress on rock failure. Journal of Geophysical Research, 72(20), 5117-5131.

Niandou, H., Shao, J. F., Henry, J. P., \& Fourmaintraux, D. (1997). Laboratory investigation of the mechanical behaviour of Tournemire shale. International Journal of Rock Mechanics and Mining Sciences, 34(1), 3-16. 
802

803

804

805

806

807

808

809

810

Nova, R. (1980). The failure of transversely isotropic rocks in triaxial compression. In International Journal of Rock Mechanics and Mining Sciences \& Geomechanics Abstracts (Vol. 17, No. 6, pp. 325-332). Pergamon.

Nova, R., and Zaninetti, A. (1990). An investigation into the tensile behavior of a schistose rock, Int. J. Rock Mech. Min. Sci., 27, 231-242.

Pollard, D., \& Aydin, A. (1988). Progress in understanding jointing over the past century, Geological Society of America Bulletin, 100, 1181-1204.

Rawling, G. C., Baud, P. and Wong, T. (2002). Dilatancy, brittle strength, and anisotropy of foliated rocks: Experimental deformation and micromechanical modeling, J. Geophys. Res., 107(B10), 2234, doi:10.1029/2001JB000472.

Renard, F., Mair, K., \& Gundersen, O. (2012). Surface roughness evolution on experimentally simulated faults. Journal of Structural Geology, 45, 101-112, doi:10.1016/j.jsg.2012.03.009.

Renard, F., Weiss, J., Mathiesen, J., Ben Zion, Y., Kandula, N., \& Cordonnier, B. (2018). Critical evolution of damage toward system-size failure in crystalline rock. Journal of Geophysical Research: Solid Earth, 123, https://doi.org/10.1002/2017JB014964.

Shea, W. T., and Kronenberg, A.K., (1993). Strength and anisotropy of foliated rocks with varied mica contents, J. Struct. Geol., 15, 1097-1121.

Scholtès, L. U. C., \& Donzé, F. V. (2012). Modelling progressive failure in fractured rock masses using a 3D discrete element method. International Journal of Rock Mechanics and Mining Sciences, 52, 18-30.

Scholtès, L., \& Donzé, F. V. (2013). A DEM model for soft and hard rocks: role of grain interlocking on strength. Journal of the Mechanics and Physics of Solids, 61(2), 352-369.

Schöpfer, M. P., Abe, S., Childs, C., \& Walsh, J. J. (2009). The impact of porosity and crack density on the elasticity, strength and friction of cohesive granular materials: insights from DEM modelling. International Journal of Rock Mechanics and Mining Sciences, 46(2), 250-261.

Smith, B., \& Sandwell, D. (2003). Coulomb stress accumulation along the San Andreas Fault system. J. of Geophysical Research: Solid Earth, 108(B6), doi:10.1029/2002JB002136. 
831 Stanchits, S., Vinciguerra, S. \& Dresen, G. (2006). Ultrasonic velocities, acoustic emission 832 characteristics and crack damage of basalt and granite, Pure Appl. Geophys., 163, $975-$ 833994.

834 Tavallali, A., and Vervoort, A., (2010). Effect of layer orientation on the failure of layered 835 sandstone under Brazilian test conditions. International Journal of Rock Mechanics \& $836 \quad$ Mining Sciences, 47, 313-322.

837 Tse, R., \& Cruden, D. M. (1979). Estimating joint roughness coefficients. In International 838 journal of rock mechanics and mining sciences \& geomechanics abstracts (Vol. 16, No. 839 5, pp. 303-307). Pergamon.

840 Weatherley, D. K., Boros, V. E., Hancock, W. R., \& Abe, S. (2010). Scaling benchmark of 841 ESyS-Particle for elastic wave propagation simulations. In e-Science (e-Science), 2010 842 IEEE Sixth International Conference on (pp. 277-283). IEEE. 


\section{$844 \quad$ Figure 1}

845 Models of blocks with all tested layer orientations, $\theta$, defined as the orientation of the normal to

846 the layer interface plane with respect to $\sigma_{1}$. Length and width of blocks are $20 \mathrm{~mm}$, and height is

$84740 \mathrm{~mm}$. Layers are $5 \mathrm{~mm}$ thick. $\sigma_{1}$ is vertical, $\sigma_{2}$ and $\sigma_{3}$ are horizontal, and $\sigma_{2}=\sigma_{3}$. Geometries

848 shown here have $0.5 \mathrm{~mm}$ overlap between layers. Colors highlight the distinct layers and the

849 orientation of preexisting weaknesses that separate each layer. All layers have identical bulk

850 strength. See Fig. S1 for details of the particle packing technique.
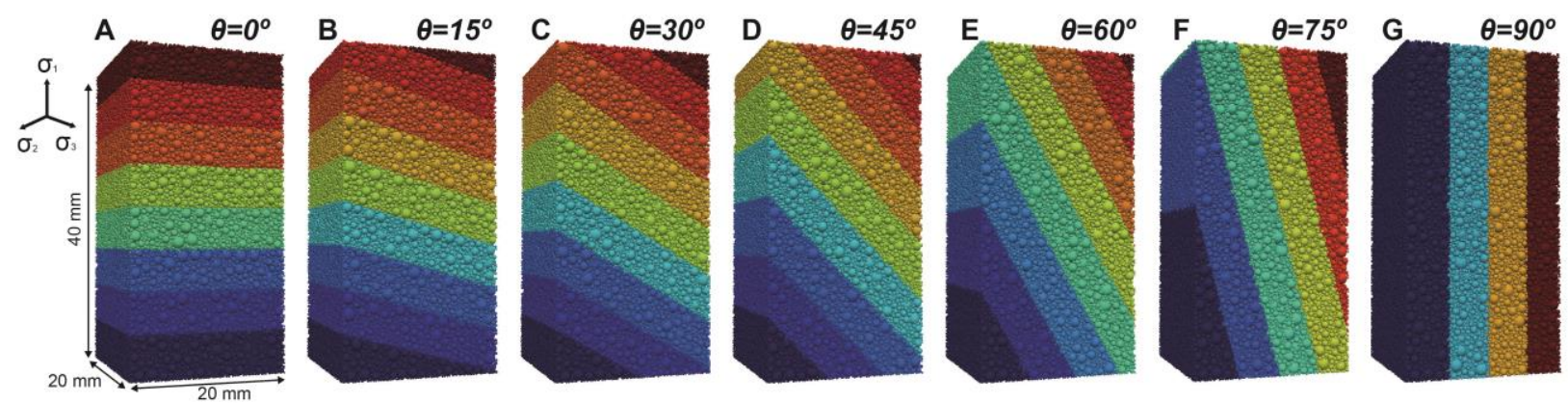


\section{$853 \quad$ Figure 2}

854 Surface topography of layer interfaces with $0.2 \mathrm{~mm}$ overlap (A-B), and $0.5 \mathrm{~mm}$ overlap (C-D) for

855 block model with layers orientated $45^{\circ}$. A, C) Topography of bottom surface of layer interface. B,

856 D) Topography of top surface of layer interface. We measure the topography as the distance

857 between particle surfaces and a basal plane at the same orientation of the layers. The bottom

858 surfaces are smoother, with lower root-mean-squared roughness, $\chi_{\text {RMs }}$, because we pack layers

859 starting from the bottom layer and work upward. With smaller overlap $(0.2 \mathrm{~mm})$, the layer

860 interfaces are smoother $\left(\chi_{\mathrm{RMS}}=0.028\right.$ for the bottom surface $)$ than with larger overlap $(0.5 \mathrm{~mm}$,

$861 \chi_{\mathrm{RMS}}=0.055$ for the bottom surface). Each topography coloring scheme ranges from the median of

862 the topography field $\pm 0.1 \mathrm{~mm}$.

863

864
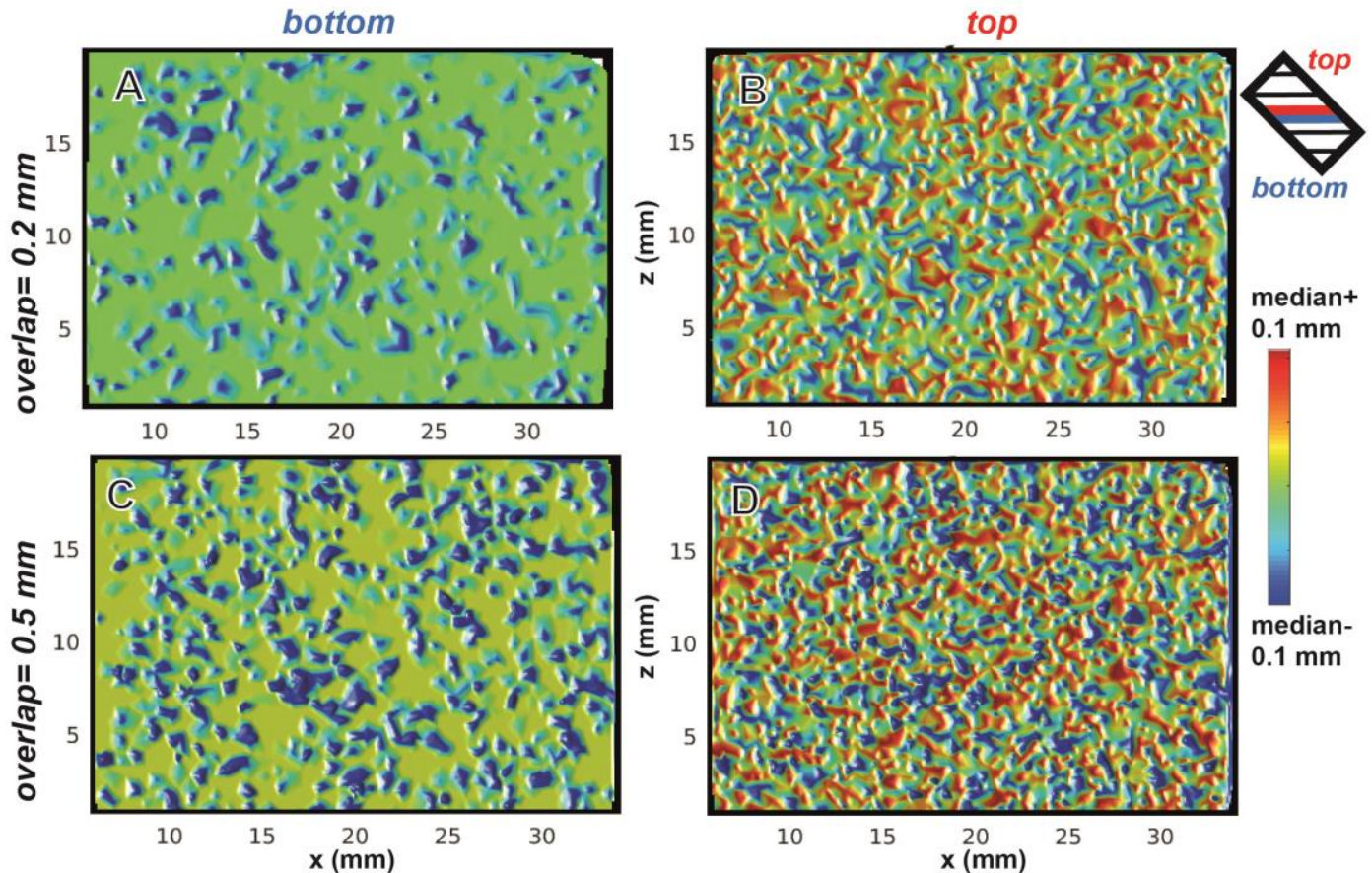

median-

$0.1 \mathrm{~mm}$ 


\section{Figure 3}

866 Mechanical loading behavior for numerical triaxial compression simulations. Results from block

867 model with layers oriented at $\theta=90^{\circ}$ and with rougher layer interfaces $(0.5 \mathrm{~mm}$ overlap) are shown.

868 A) Axial contraction versus differential stress for confining stresses 0-50 MPa. Squares show

869 failure stress, $\sigma_{\mathrm{F}}$, i.e., the peak stress preceding failure and the first stress drop, and vertical lines

870 show axial contraction at failure. B) Resulting relationship between applied confining stress, $\sigma_{2}$,

871 and $\sigma_{F}$ including the linear regression from which we estimate $\mu_{0}$. The slope of the failure envelope

872 of $\sigma_{2}$ and $\sigma_{\mathrm{F}}$ decreases with increasing $\sigma_{2}$, and so is slightly non-linear.
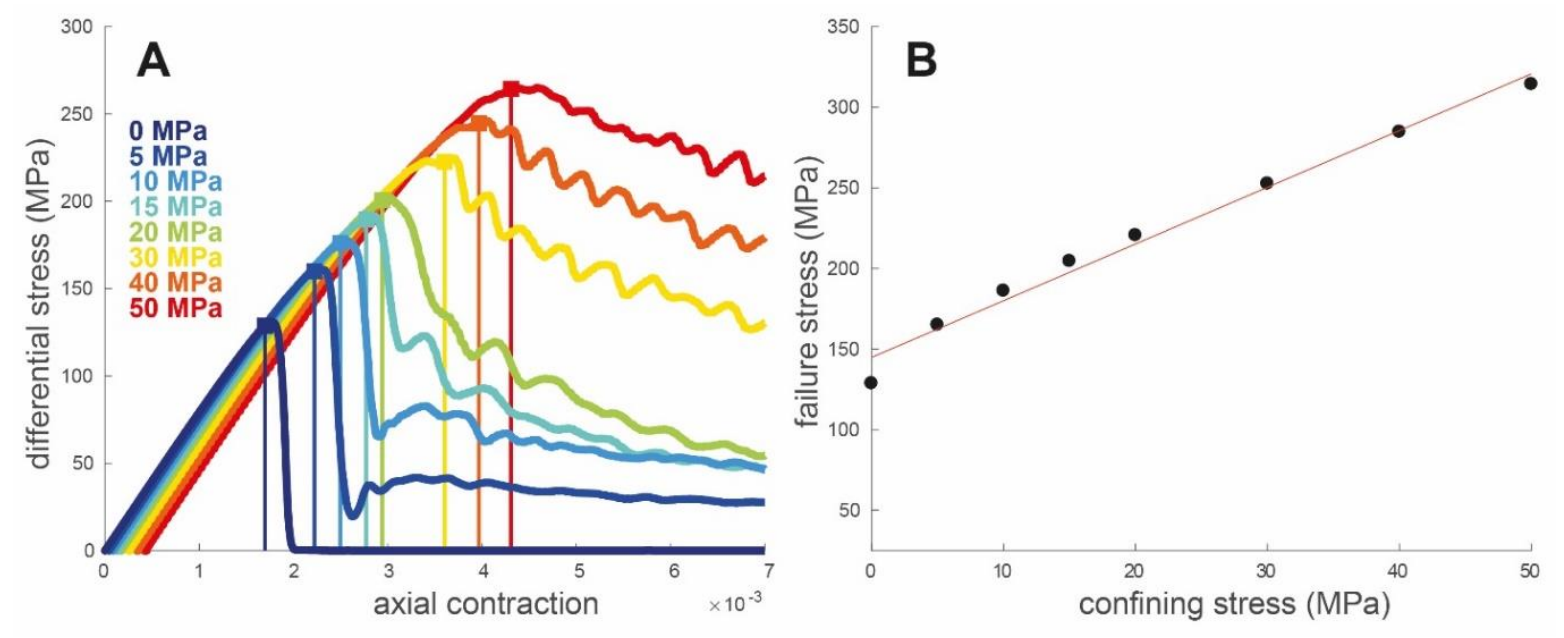


\section{Figure 4}

876 Distribution of $\sigma_{\mathrm{F}}$ as a function of $\sigma_{2}$ and $\theta$ for $(\mathrm{A}, \mathrm{B})$ smoother layer interfaces $(0.2$ mm overlap)

877 and (C, D) rougher layer interfaces (0.5 mm overlap), and (A, C) cube and (B, D) block geometries.

878 Results from lower (0-20 MPa), median (20 MPa), and higher (20-50 MPa) $\sigma_{2}$ ranges are shown

879 in red, black, and blue, respectively. Triangles show results from homogeneous block model that 880 does not contain layers.

881

882
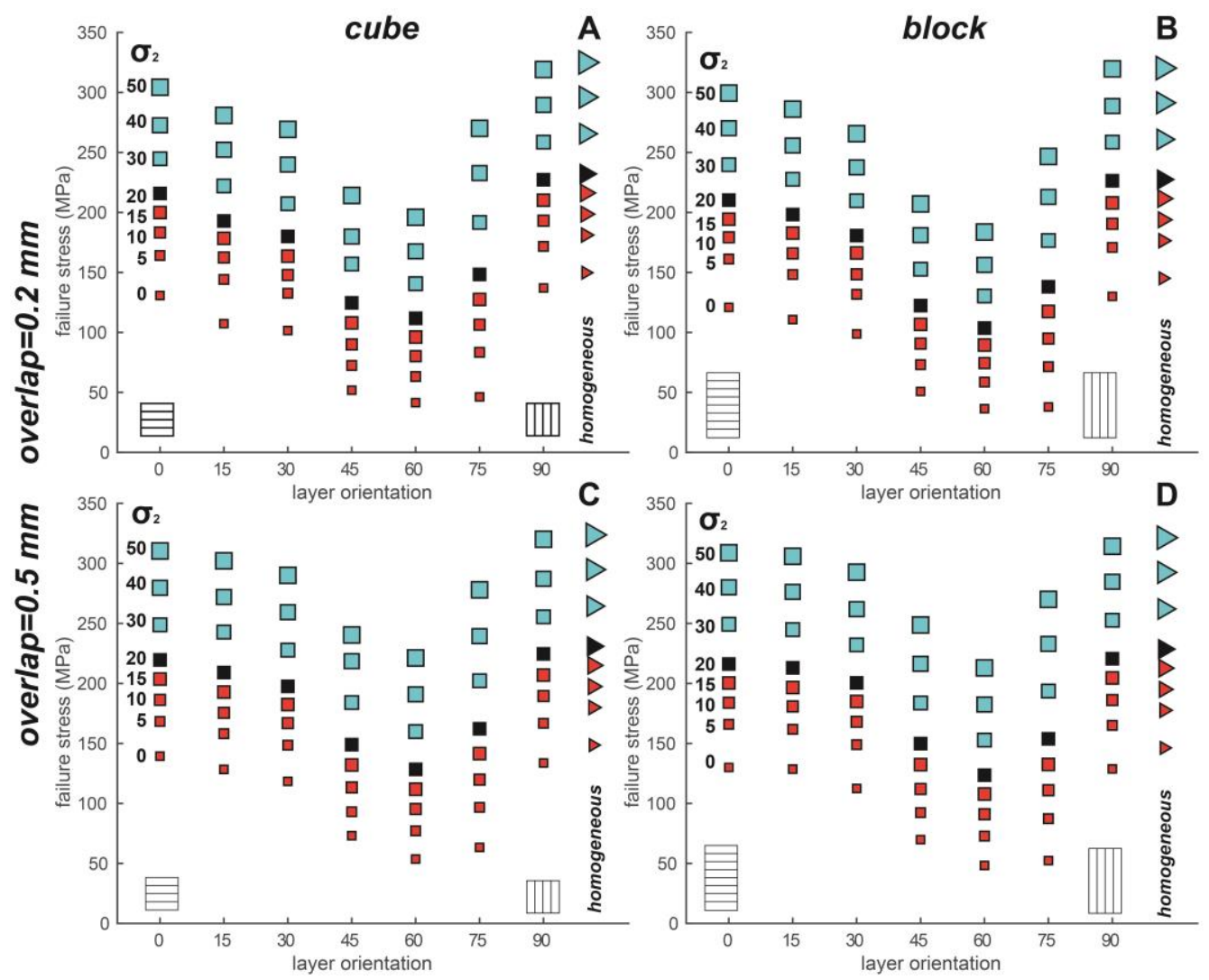


\section{$883 \quad$ Figure 5}

884 Distribution of $\mu_{0}$ as a function of $\sigma_{2}$ and $\theta$, for $(A, B)$ smoother layer interfaces $(0.2$ mm overlap)

885 and (C, D) rougher layer interfaces (0.5 mm overlap), and (A, C) cube and (B, D) block geometries.

$886 \mu_{0}$ calculated from higher (20-50 MPa), lower (0-20 MPa), and full (0-50 MPa) $\sigma_{2}$ range shown in

887 blue, red and black, respectively. Horizontal lines show results from homogeneous block model

888 that does not contain layers.

889

890

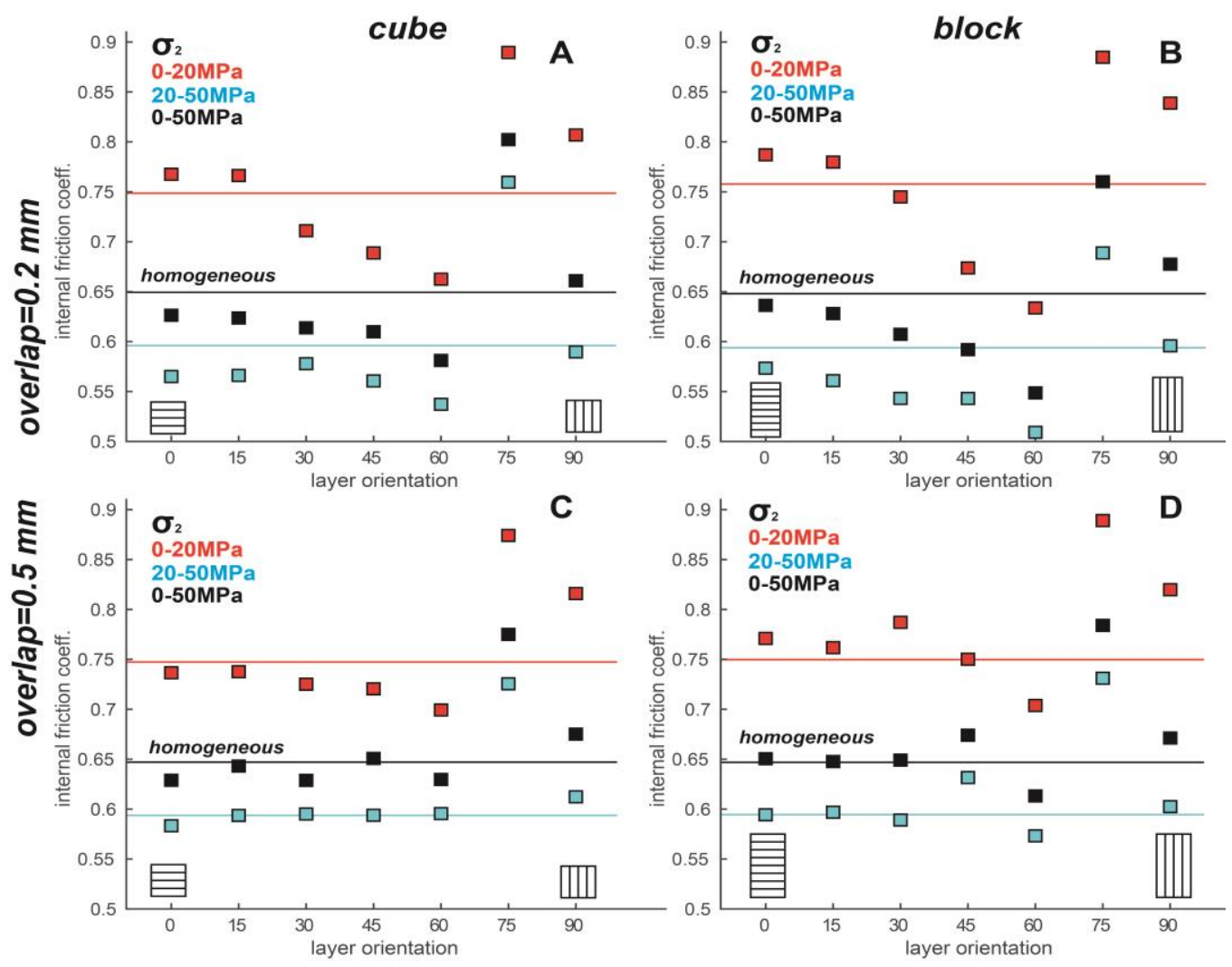


892 Relative impact of parameters on failure: (A) interface orientation, (B) interface roughness, (C)

893 interface area (i.e., model shape). D) Difference in maximum and minimum $\mu_{0}$ of each set of

894 models comparing area $(\mathrm{C})$ and roughness $(\mathrm{B})$, and the difference in $\mu_{0}$ when $\theta=90^{\circ}$ and the

895 minimum $\mu_{0}(\mathrm{~A})$. A) Color of symbol indicates layer orientation. B) Results from block models

896 with $0.5 \mathrm{~mm}$ overlap (black) and $0.2 \mathrm{~mm}$ overlap (white). C) Results from models with $0.2 \mathrm{~mm}$

897 overlap with cube shapes (squares) and block shapes (triangle). D) Impact of parameters on the

898 difference in $\mu_{0}$. Varying $\theta$ produces the largest $\Delta \mu_{0}$, even discounting when $\theta=75^{\circ}$. Varying the

899 layer interface area (model shape) produces the smallest $\Delta \mu_{0}$. Fig. S8 compares results from all of

900 the models.

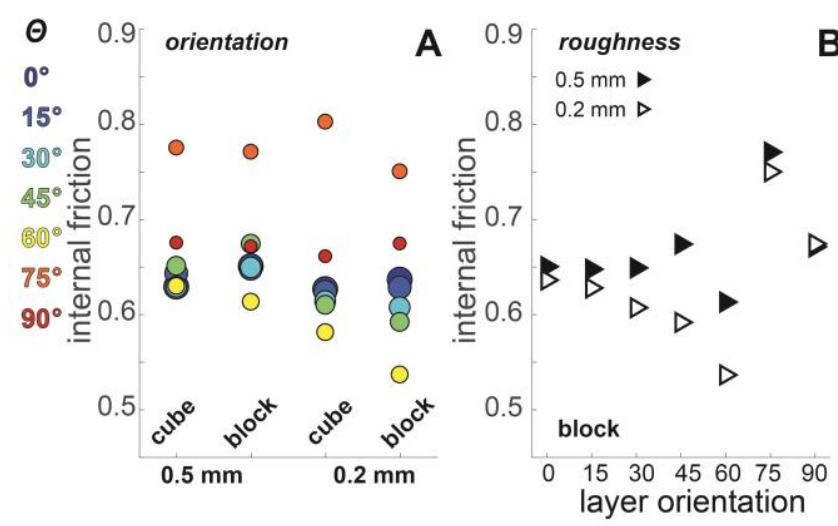

901 


\section{$902 \quad$ Figure 7}

903 Spatial distributions of particle velocities

904 highlight the influence of $\theta$ and $\sigma_{2}$ on strain

905 distribution along interfaces and within host.
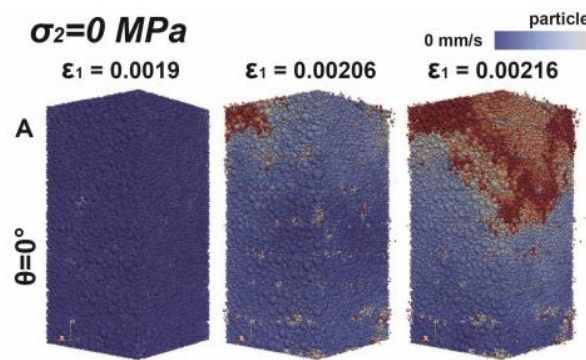

particle velocity $15 \mathrm{~mm} / \mathrm{s}$

$\varepsilon_{1}=0.0003$

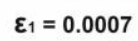

$\varepsilon_{1}=0.0008$

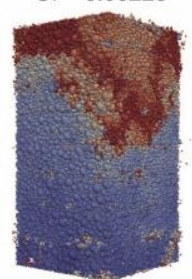

906 Failure of simulated layered rock with $\sigma_{2}=0$

$907 \mathrm{MPa}$, and (A) $\theta=0^{\circ}$, (B) $\theta=60^{\circ}$, and (C) $\theta=90^{\circ}$,

908 and with $\sigma_{2}=10 \mathrm{MPa}$ and (E) $\theta=0^{\circ}$, (F) $\theta=60^{\circ}$,

909 and (G) $\theta=90^{\circ}$. Black numbers are the

B
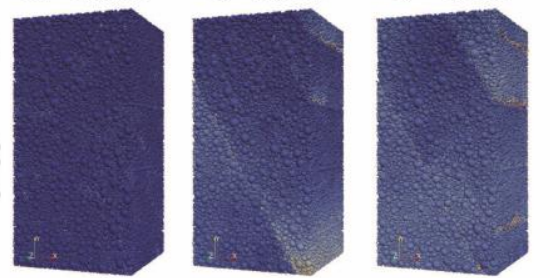

$\varepsilon_{1}=0.0018$

$\varepsilon_{1}=0.0019$
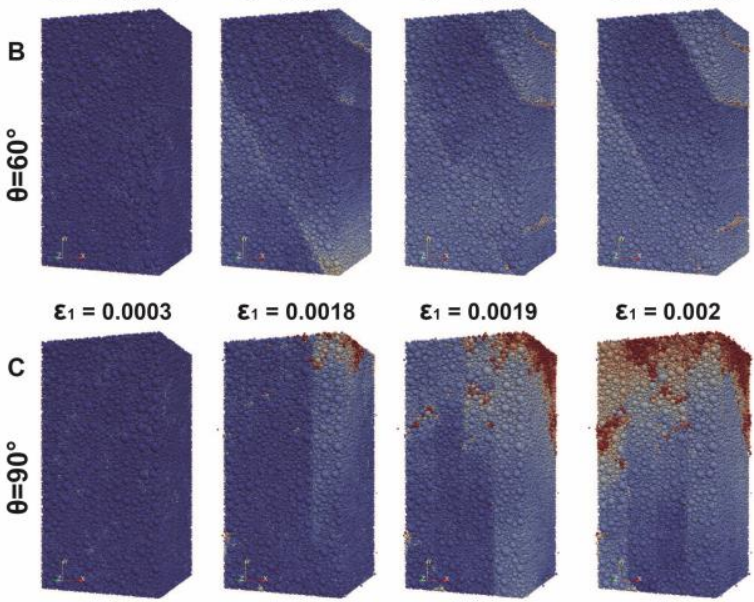

$\varepsilon_{1}=0.002$

910 accumulated axial strain, which increase toward

911 the right. Colors show the total magnitude of the

912 velocity of particles. Block models with $0.2 \mathrm{~mm}$

913 overlap are shown.

914
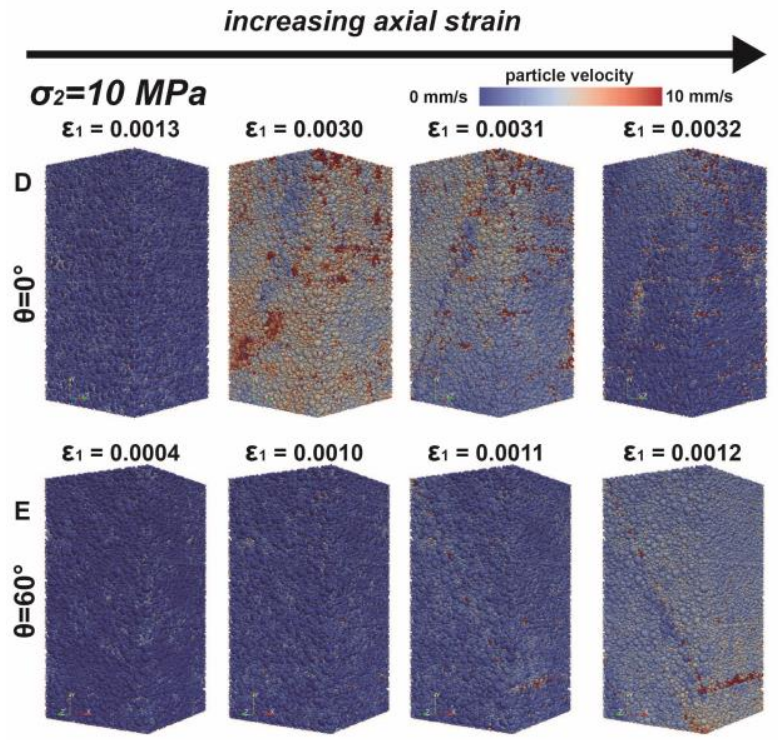

$\varepsilon_{1}=0.0012$

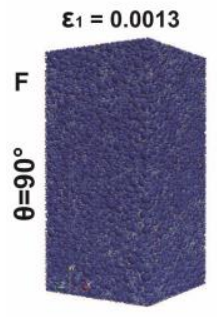

$\varepsilon_{1}=0.0027$

$\varepsilon_{1}=0.0028$
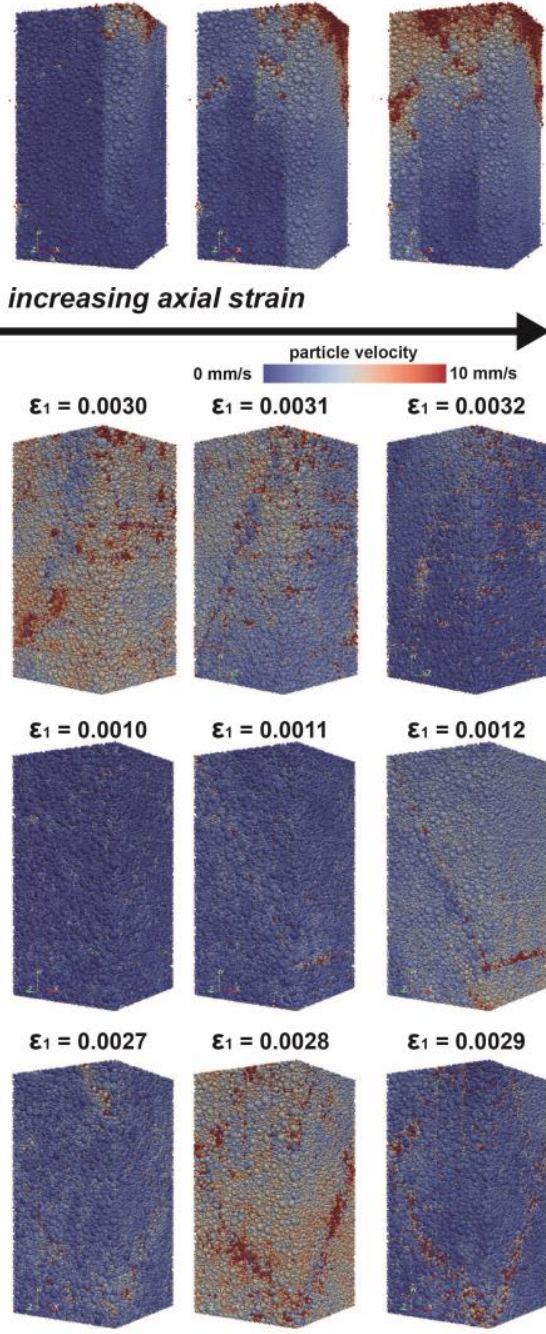

$\varepsilon_{1}=0.0029$

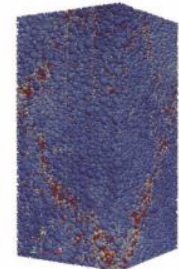




\section{$915 \quad$ Figure 8}

916 Spatial distributions of nucleating fractures

917 highlight the influence of $\theta$ and $\sigma_{2}$. Failure of

918 simulated layered rock with $\sigma_{2}=0 \mathrm{MPa}$, and (A)

$919 \theta=0^{\circ},(\mathrm{B}) \theta=60^{\circ}$, and (C) $\theta=90^{\circ}$, and with $\sigma_{2}=10$

$920 \mathrm{MPa}$ and (D) $\theta=0^{\circ}$, (E) $\theta=60^{\circ}$, and (F) $\theta=90^{\circ}$.

921 Black numbers are the accumulated axial strain.

922 Black lines show location of bonds that break at

923 each time interval, i.e., nucleating fractures. Block

924 models with $0.2 \mathrm{~mm}$ overlap are shown.
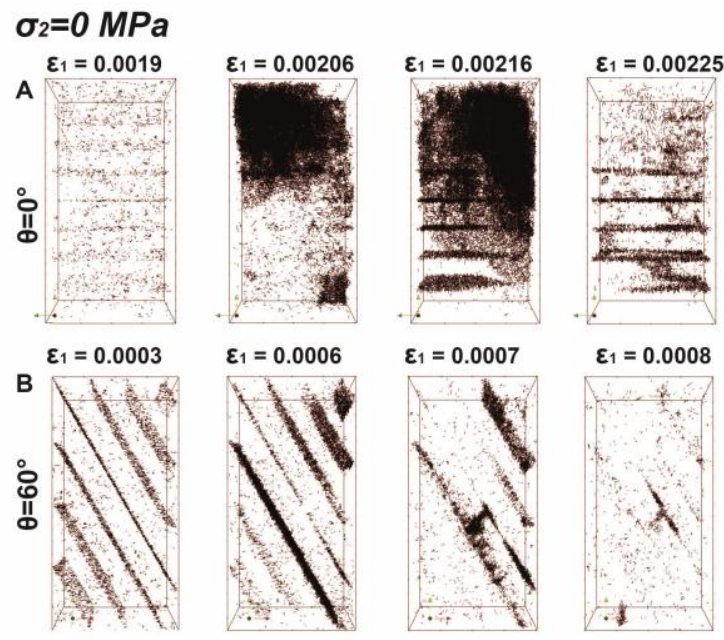

$\varepsilon_{1}=0.0006$

$\varepsilon_{1}=0.0007$
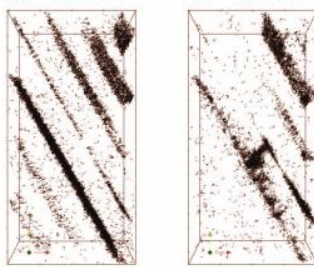

$\varepsilon_{1}=0.0008$
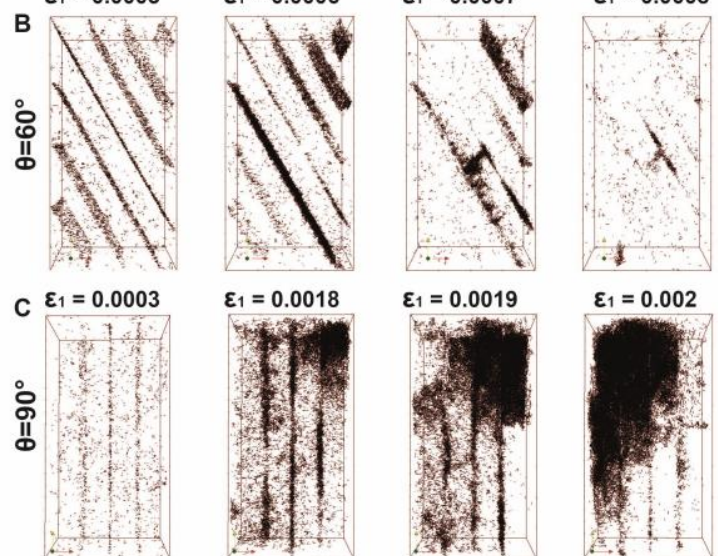

$\varepsilon_{1}=0.0018$

$\varepsilon_{1}=0.0019$
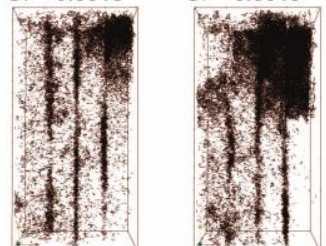

$\varepsilon_{1}=0.002$

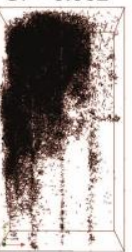

increasing axial strain
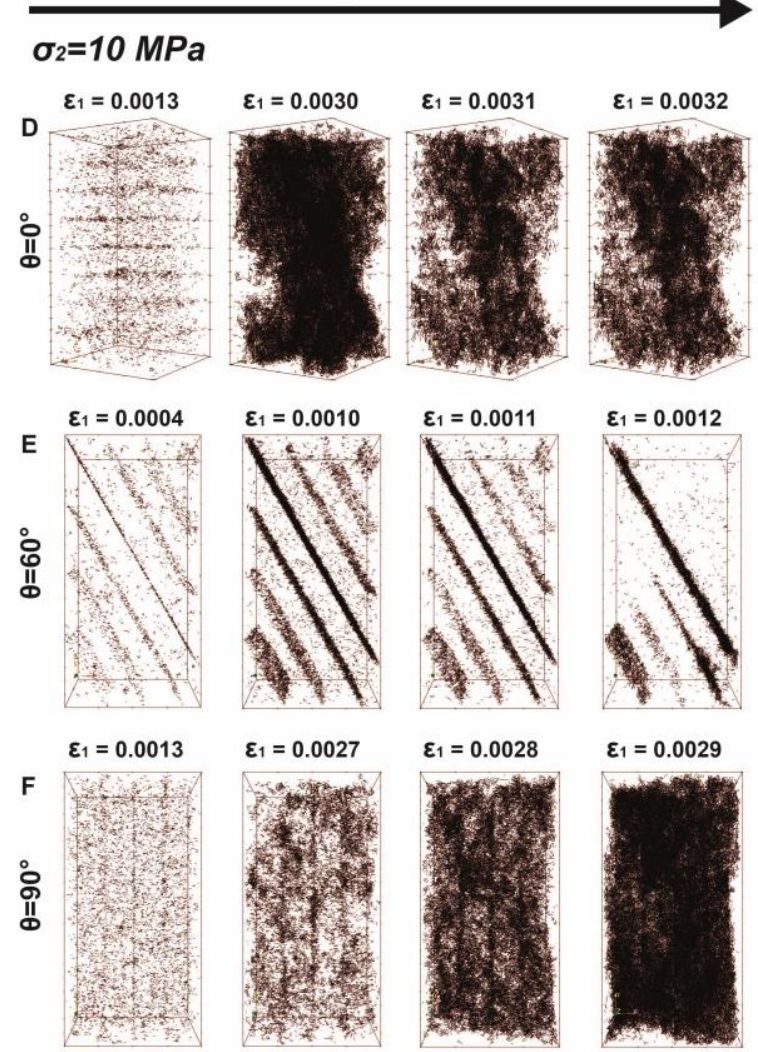
927 Evolution of failure, characterized as $\Delta \mathrm{B}$, indicates the relative impact of fracturing along weak

928 interfaces compared to within the host rock. Failure localization, $\Delta \mathrm{B}$, is shown as the difference

929 between the percentage of weak bonds broken out of all the weak bonds, and the percentage of the

930 strong bonds broken out of all the strong bonds for a representative range of $\sigma_{2}:(\mathrm{A}) \sigma_{2}=0 \mathrm{MPa}$,

931 (B) $\sigma_{2}=20 \mathrm{MPa}$, and $(\mathrm{C}) \sigma_{2}=50 \mathrm{MPa}$ with all tested layer orientations, $\theta$, and for a representative

932 range of $\theta$ : (D) $\theta=0^{\circ},(\mathrm{E}) \theta=60^{\circ},(\mathrm{F}) \theta=90^{\circ}$, and all tested $\sigma_{2}$. A-C) Color of lines correspond to $\theta$.

933 D-F) Color of lines correspond to $\sigma_{2}$. Data are presented for smoother layer interfaces $(0.2 \mathrm{~mm}$

934 overlap) of block models.
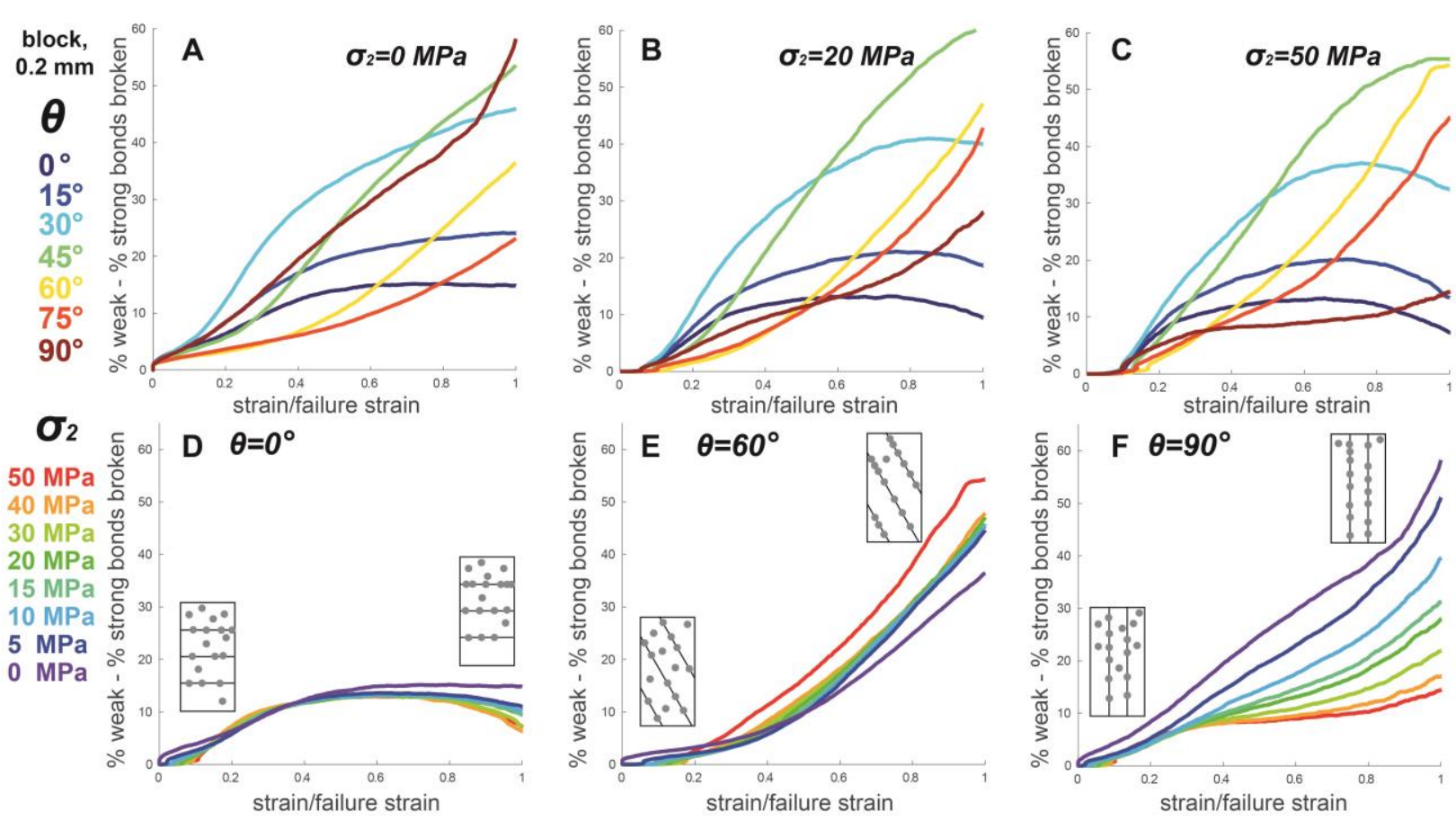


\section{Figure 10}

938 Evolution of anisotropic ratio, $\mathrm{K}_{2}$, for models with varying layer interface roughness and area 939 available for slip. Following Lisjak et al. (2014), $\mathrm{K}_{2}$ is the ratio between the maximum and

940 minimum differential stress at failure for experiments with different layer orientations, and

941 constant confining stress (Eq. 3). Models with smoother layers (0.2 mm overlap, red and yellow

942 squares $)$ behave more anisotropically than models with rougher layers ( $0.5 \mathrm{~mm}$ overlap, light and 943 dark blue squares). Models with block shapes (red and dark blue squares) behave more

944 anisotropically than models with cube shapes (yellow and light blue squares), as expected from

945 the larger difference in area available for slip in block models. Increasing confining stress 946 suppresses anisotropic behavior.

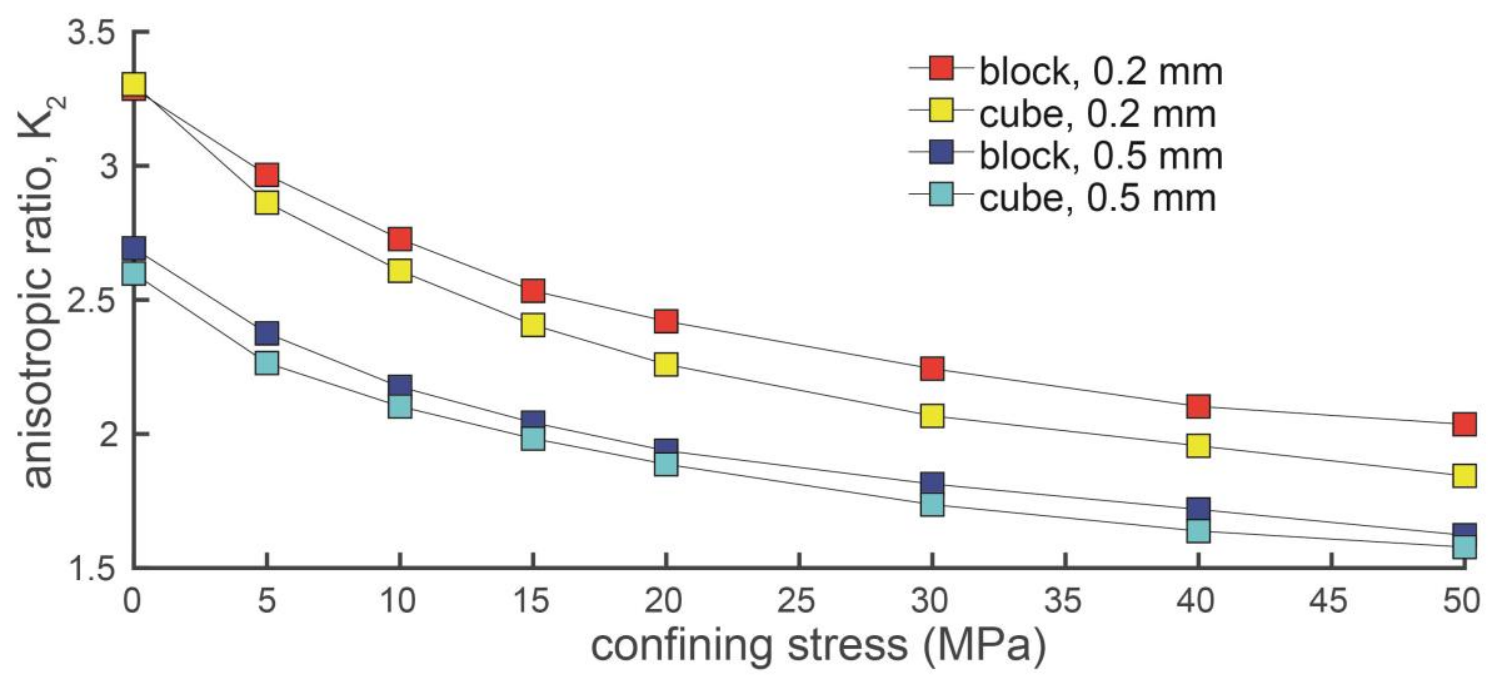




\section{$947 \quad$ Figure 11}

948 Sketch summarizing the relative importance of preexisting weakness property on the macroscopic

$949 \mu_{0}$. Preexisting weaknesses produce greater changes in $\mu_{0}$ at lower confining stress, at intermediate

950 layer orientations relative to $\sigma_{1}$, with

951 smoother layer interfaces, and with

$$
<1 \mathrm{~km} \quad \text { decreasing } \mu_{0}
$$

$>1 \mathrm{~km}$ increasing $\Delta \mu_{0}$

952 greater total layer interface area.

953 Varying the orientation produces the

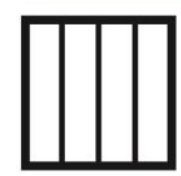

954 largest changes in $\mu_{0}$, and varying the

955 interface area produces the smallest

956 changes in $\mu_{0}$ over the range of

957 parameter space explored here.
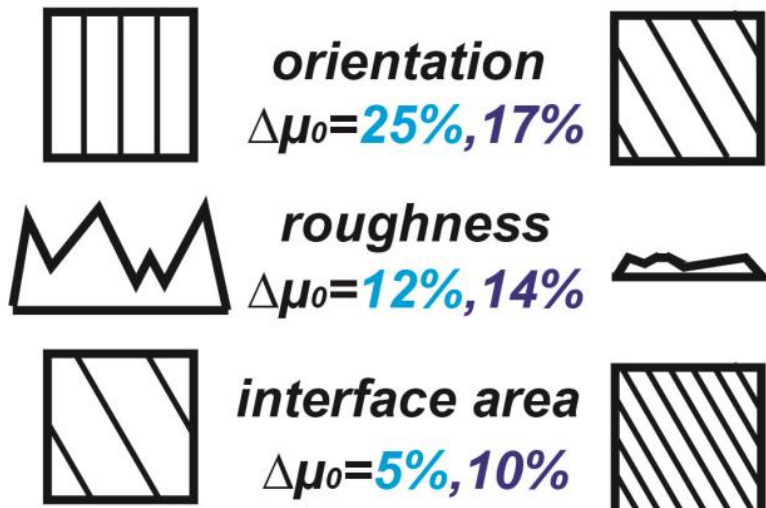

roughness

$\Delta \mu_{0}=12 \%, 14 \%$

interface area $\Delta \mu_{0}=5 \%, 10 \%$

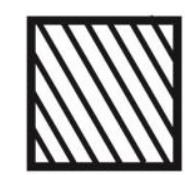

